\title{
SIGNIFICADO HISTÓRICO Y ACTUALIDAD DE LAS POLÍTICAS DE REDUCCIÓN DEL TIEMPO DE TRABAJO ${ }^{1}$
}

\author{
Mikel de la Fuente Lavín \\ Jon Bernat Zubiri Rey \\ Euskal Herriko Unibertsitatea/Universidad del País Vasco (UPV/EHU)
}

DOI: $10.1387 /$ lan-harremanak. 16554

\section{ABSTRACT}

En este artículo señalaremos algunos elementos de diagnostico sobre el significado histórico y la actualidad de las politicas Reducción del Tiempo de Trabajo (RTT), en un contexto de crecimiento económico cada vez menos generador de empleos y su consecuente paro estructural. Se abordará, también, la cuestión del empleo a tiempo parcial forzado, generador de una dualidad contraria a la RTT generalizada. Por último analizaremos algunas cuestiones, dificultades y ventajas de la puesta en práctica de politicas de RTT. De lo expuesto se extrae que una implantación de una RTT generalizada sin reducción de salarios debe permitir la recuperación de la pérdida de la parte salarial que en los últimos decenios ha ido a los beneficios empresariales. La RTT sustancial, equitativa y redistributiva constituye una alternativa al reparto del trabajo a través del desarrollo del tiempo parcial, que en los casos vasco y español es mayoritariamente involuntario y mantiene una división sexual del trabajo en perjuicio de las mujeres.

Palabras clave: tiempo, reducción, trabajo, reparto, empleo.

${ }^{1}$ La autoría de este artículo está indicada por orden alfabético, justificada en el trabajo equitativamente compartido de análisis del reparto del tiempo de trabajo, en el marco del Proyecto de Investigación «Reparto del empleo y del tiempo de trabajo: efectos en la fiscalidad y tratamiento jurídico», financiado por el Departamento de Innovación de la Diputación Foral de Gipuzkoa, a ejecutar entre el 22-12-2014 y el 30-10-2015. 
In this article we will point some elements about the historical significance and currently of working-time reduction (WTR), in a context of economic growth being less and less able to generate employments and the consequent structural unemployment. We will focus, as well, on the part-time work, a cause of duality opposed to the generalized WTR. At the end, we will analyze some questions, difficulties and advantages of the WTR politics. Our explanation arguments about the need of a generalized WTR without any wage cuts. This way should allow workers to recover the part of revenues has been appropriated by entrepreneurial profits in the last decades. The relevant, equitable and redistributive WTR is an alternative to the share of work by the development of part-time employment, being in Spanish and Basque cases mostly involuntary and perpetuating against women the sexual division of the work.

Keywords: time, reduction, work, share, employment.

Lanaldi murrizketa (LM) politiken garrantzia historikoa eta gaurkotasunari buruzko diagnosi elementuak aurkeztuko ditugu artikulu honetan, lanpostu gutxiago sortzeko gaitasuna duen hazkunde ekonomikoa eta ondorioz egiturazkoa den langabezi testuinguruan. Behartutako denboraldi partzialeko enplegua ere aztertuko dugu, LM orokortuaren aurkako dualitatearen iturria dena. Bukatzeko, LM politikak egitearen zenbait alde, arazo eta onura aztertuko ditugu. LM orokortua, soldata gutxitu barik egiten den heinean, azken hamarkadetan enpresen etekinetara bideratu den soldaten atala berreskuratzeko erabilgarria da. Esanguratsua, ekitatiboa eta birbanatzailea den LM-a denboraldi partziala garatzen egindako lan banaketaren alternatiba da. Espainiar eta euskal kasuetan bereziki behartuak izaten dira hauek, emakumeen kalterako, lanaren sexu banaketa egonkortuz.

Hitz gakoak: denbora, murrizketa, lana, banaketa, enplegua. 


\section{Introducción}

La reducción de las extenuantes y larguísimas jornadas de la Revolución Industrial fue uno de los primeros objetivos del movimiento obrero y el naciente sindicalismo. Las normas que progresivamente la fueron poniendo en práctica fueron constituyentes, a finales del siglo xIx y principios del siglo xx, del Derecho del Trabajo a escala estatal y, posteriormente, internacional. En un primer momento las medidas de reducción de la jornada se inspiran en la necesidad de mejorar la seguridad y salud de los trabajadores. El Tratado de Versalles que ponía fin a la Primera Guerra Mundial recomendaba la jornada semanal de 8 horas diarias, 48 semanales y que los domingos fuesen festivos, propuestas que fueron recogidas por la Organización Internacional del Trabajo (OIT) en 1919, con el añadido de que se librara la tarde de los sábados. La atribución a la reducción del tiempo de trabajo (en adelante, RTT) del objetivo de la creación de empleo se propuso por primera vez en Estados Unidos con motivo de la «Gran Depresión» de 1929. Aunque el Senado llegó a aprobar una ley que fijaba una jornada máxima de 30 horas semanales, las presiones de la patronal llevaron a que el presidente Roosevelt derogase la ley (Imaz Bengoetxea, 2006: 9 ; 2003).

La limitación de las horas de trabajo se ha expresado de forma mayoritaria mediante la fijación de límites a la jornada semanal, como se acredita por el gran número de países que han reducido la jornada semanal de 48 a 40 horas en las últimas décadas. Así, en el año 2009 el 41\% de los Estados tiene establecida la jornada de 40 horas semanales, entre ellos el $67 \%$ de los países desarrollados y de la Unión Europea. Del 44\% cuya jornada regular supera las 40 horas, más de la mitad ha fijado un límite de 48 horas (OIT, 2011: 14-15).

En España, en 1913 la jornada laboral sobrepasaba en algunos sectores productivos las 3.000 horas anuales. Tras una huelga en el sector textil de Barcelona, el gobierno español aprobó un decreto que fijaba la jornada en 60 horas semanales y 3.000 anuales, medida a la que se opuso la patronal del sector, que pidió su suspensión. Por Decreto de 3 de abril de 1919 se aprobó la jornada de 8 horas diarias y 48 a la semana, que hasta esa fecha sólo era realizada por el $17,8 \%$ de los obreros, que fue incrementándose progresivamente pero con un elevado número de infracciones por las empresas, constatadas por los inspectores de trabajo (Soto Carmona, 1989: 612-615). Entre los años 1919 y 2014, es de- 
cir, en 95 años, la jornada legal se ha reducido en España solamente ocho horas por semana, lo que equivale a reducciones de cinco minutos cada año durante todo el periodo (García Ninet, 2000: 6). Que en ese mismo período se haya producido un enorme incremento de la producción y de la productividad, es una buena razón para mantener que los obstáculos para reducir sustancialmente el tiempo de trabajo no están en la economía sino fuera de ella: para producir lo que 100 personas en la España de 1960, se necesitaban en 1995 únicamente 27 personas (Montes, 1996: 82) y 24,4 en $2012^{2}$. Obviamente la reducción del tiempo de trabajo no ha seguido, ni de lejos, esa evolución, pero el motivo está en las relaciones sociales y políticas.

La actual negativa por parte de las principales asociaciones patronales a reducir la jornada de trabajo enlaza con su oposición histórica a la reducción de las jornadas de 14 y más horas en el siglo xIX y a la implantación de la jornada de 40 horas en el presente siglo. Por ejemplo, la persistente negativa del Reino Unido para asumir las obligaciones establecidas en la normativa de la Unión Europea (UE) de reducción de la jornada semanal a cuarenta y ocho horas y su oposición frontal a la supresión de la cláusula opt-out. En efecto, Gilbert (1998) señala que:

Todas las intervenciones públicas que han pretendido disminuir la duración del trabajo (quitando las que se limitan a meras incitaciones financieras) a lo largo de una semana (por ejemplo reduciendo las duraciones máximas), un año (instauración y desarrollo de bajas pagadas) o de todo el ciclo vital (prohibición de trabajo infantil, alargamiento de la escolaridad obligatoria...) se han enfrentado a la oposición viva y a veces violenta de una gran parte de la patronal pero también de otros muchos participantes del debate público.

Esto no ha impedido que, durante las últimas décadas, haya tenido lugar una cierta reducción de la jornada semanal de trabajo en la Unión Europea, desde las 40,5 horas de la UE 12 en 1991 a las 37,5 horas en el 2010 de la UE 27 (36,4 para la UE 12), que se debe a los siguientes cuatro factores (Eurofound, 2010: 4):

— La proporción de la fuerza de trabajo que realiza largas jornadas (más de 48 horas por semana) se ha reducido: en la UE 27 desde el 15\% en 2000 a $12 \%$ en 2010 . Las jornadas amplias permanecen, en lo esencial, un fenómeno masculino: en el 2007 eran realizadas por el 18\% de los hombres frente a un $8 \%$ de las mujeres. Son también una característica de los trabajadores por cuenta propia, ya que el $42 \%$ de los mismos trabajaban más de 48 horas a la semana. También se realizan en proporción más ele-

\footnotetext{
2 Cálculos realizados — manteniendo la metodología de Montes (1996) — en el marco de la investigación sobre necesidad y viabilidad de las políticas de RTT (Zendoia et al., 2015)
} 
vada en la industria manufacturera (el 20\% trabajaba más de 48 horas en el 2010) que en los servicios (el 15\%), aunque la tendencia a la reducción es paralela en ambos sectores.

- La proporción de fuerza de trabajo con jornadas más cortas (menos de 20 horas por semana) ha aumentado, desde el $8 \%$ en 1991 en la UE 12, hasta el 14\% en 2010. En la UE 27 ha pasado desde el $12 \%$ en el 2000 hasta el $13 \%$ en el 2010.

- Los acuerdos colectivos sobre tiempo de trabajo han establecido reducciones de jornada en algunos países y sectores.

- Las reducciones en la jornada de trabajo como reacciones a la recesión iniciada en el 2008.

Sin embargo, en los últimos años está teniendo lugar un aumento de la jornada a de los trabajadores a tiempo completo en varios países europeos, que es muy probable que se extienda «por contagio» al conjunto de los mismos. Así, según Pradella (2015):

Alemania ha vuelto al nivel anterior a la crisis de poco menos de 41 horas semanales, mientras que el Reino Unido asiste al retorno de la «cultura de las horas largas», pues mientras casi uno de cinco ocupados percibe un salario bajo, un quinto de los ocupados a jornada completa trabajan regularmente más de 45 horas a la semana. En Italia, el porcentaje de ocupados a jornada completa que trabajan más de 45 horas a la semana (un 16,3\% en 2011) casi se ha duplicado desde 2002.

La reducción de la jornada ordinaria citada se acompaña con el mantenimiento de un nivel elevado de realización de horas extraordinarias. Según la OIT, en la UE cerca de dos terceras partes de los establecimientos productivos recurren a horas extraordinarias cada año y casi la mitad de los asalariados realizan esas horas. En la UE el 35\% de las empresas compensan el horario extraordinario mediante remuneración, el 23\% a través de tiempo libre (lo que constituye una manifestación de jornada irregular), un 37\% utiliza ambas formas de remuneración y un $4 \%$ no se compensa de ninguna forma, según la OIT especialmente en los establecimientos con un elevado porcentaje de trabajadores que ocupan puestos «altamente calificados» (Oficina Internacional del Trabajo, 2011: 46).

Estas estadísticas de la OIT no parecen creíbles, ya que en la UE la precarización e inseguridad del trabajo entraña un incumplimiento de la normativa laboral, en este caso de la obligación de que las horas extraordinarias deben ser compensadas o pagadas. En España, según un informe sindical (UGT, 2015), aunque a lo largo de la crisis, como consecuencia de la reducción de las plantillas, ha descendido un $41,1 \%$ el número de asalariados que realiza horas extraordinarias (594.800 personas, el 4\% del conjunto de los asalariados), el número de trabajadoras que cobra las horas extraordinarias ha caído un 57,6\% desde 2008, 
frente a una reducción del $11,6 \%$ de quienes no las cobran. El resultado es que del total de quienes efectúan horas extra el $56 \%$ — según este informe- no las cobra, mientras que en el 2008 esa proporción era del 35\%. El volumen de horas extras entre 2008 y 2014 ha caído el 40,3\% y del total de las horas extras realizadas, el 52\% no están remuneradas, 14 puntos más que en 2008 (UGT, 2015: 4-5) .

De esta situación se desprende que ese aumento de las horas extras no pagadas implica una reducción del salario/hora que se suma a la del salario ordinario. Para quienes las cobran, de investigaciones empíricas se desprende que la principal razón de su aceptación consiste en una presión consumista que llevan a extender el trabajo remunerado a través de acumulación de varios empleos o de la realización habitual de horas extraordinarias, ya que en el contexto de la actual devaluación salarial la remuneración de un solo puesto de trabajo es insuficiente para mantenerse en la norma de consumo (Callejo, 2007: 161).

La condición salarial, a la que habían ido unidas la extensión de los derechos sociales y de ciudadanía, entra en quiebra con la extensión masiva del paro, la precariedad y el recorte de los derechos sociales (Moreno, 2003; 2009). Una vez conseguidas las reivindicaciones históricas del movimiento obrero de la jornada de ocho de horas y la semana de cinco días, se frena asimismo el proceso social de reducción generalizada de la jornada de trabajo sobre el conjunto de la vida ${ }^{4}$. Este proceso se simultanea con una pérdida de la rigidez de los horarios típica de la fase fordista (Lettieri, 1999: 31), que es sustituida por un tiempo de trabajo permeable a las fluctuaciones de la demanda y a las necesidades inmediatas -en muchos casos omnipresentes a las vez que imprevisibles - de las empresas, siendo estos procesos diferenciados según los sectores productivos, las categorías profesionales y los sexos (Morini, 2014).

Cada vez más, las (débiles) reducciones de la jornada se ven acompañadas de la contrapartida flexibilizadora de su distribución irregular y de la jornada semanal. Es decir, cada vez son menos generalizadas las jornadas que consisten en un número fijo de horas de trabajo por día durante un número fijo de días, ge-

${ }^{3}$ La realidad de esta situación de horas extraordinarias no abonadas se encuentra de alguna forma confirmada por la Ministro de Trabajo que declaraba en diciembre de 2014 que de 2.900 inspecciones en relación con las horas extraordinarias se habían encontrado irregularidades en más del 60\% de los casos (El Mundo, 10-12-2014). Según datos del INE del 2. ${ }^{\circ}$ trimestre del 2015, en España se realizan 6,5 millones de horas extras a la semana, de la cuáles 3,9 millones no son pagadas - 60\% de total- Conviene recordar que el Estatuto de los Trabajadores establece una cantidad máxima de 80 horas extras anuales por persona asalariada, además de obligar a su carácter voluntario (Durán, 2015).

${ }^{4}$ Según Viard (2013) la duración del trabajo en proporción con el conjunto de vida habría pasado del $40 \%$ al $12 \%$ entre 1900 y la actualidad. El mismo autor indica que a mediados del siglo xx los hombres trabajaban de media 120000 horas a lo largo de su vida, las mismas que en la actualidad realiza una pareja doméstica. 
neralmente de lunes a viernes. Estos horarios atípicos son más frecuentes entre los trabajadores que ocupan puestos de trabajo sin exigencias específicas de formación que, a su vez, tienen menos probabilidad de ejercer un control en lo que respecta a su tiempo de trabajo. Este último factor podría contribuir a compensar los efectos negativos de estos horarios atípicos y flexibles sobre el equilibrio trabajo-vida, ya que es muy probable que incidan de forma negativa en su vida social y cívica (Oficina Internacional del Trabajo, 2011: 44 y 62). Asimismo, es muy posible también que produzcan vidas a contratiempo, agotamiento físico y moral (Lallement, 2007: 61) y mayores tensiones en el conflicto entre el tiempo de producción para el mercado y el capital y los tiempos de vida necesarios para participar en las tareas de cuidados - el conflicto capital-vida de las economistas feministas- (Orozco, 2014).

De la misma forma, ha tenido lugar un importante desarrollo del trabajo nocturno, a turnos y en fin de semana: en la Unión Europea el 18\% de los trabajadores trabaja de noche, el $17 \%$ lo hace a turnos y el $26 \%$ trabaja al menos un domingo al mes (Eurofound, 2010: 4). La reducción del tiempo de trabajo (RTT) se ha transmutado en reducción y reorganización del tiempo de trabajo (RRTT), con un peso creciente de lo segundo en relación con lo primero. Recogiendo los estudios realizados al respecto, la OIT (2011: 63) concluye que los sistemas de tiempo de trabajo flexible afectan desfavorablemente a la salud y bienestar de los trabajadores, en materias tales como la calidad del descanso nocturno. Esto sucede cuando son los empresarios quienes tienen el control de esa flexibilidad, mientras que cuando la misma corresponde a la opción de las personas asalariadas inciden favorablemente en una serie de parámetros que tienen que ver no sólo con la seguridad y la salud en el trabajo, sino también con el equilibrio conflictivo entre capital-trabajo-vida antes mencionado y la propia eficiencia y eficacia organizativas.

Por otra parte, con el desarrollo de la urbanización y el alejamiento de la residencia de los trabajadores en relación con los centros de trabajo, han aumentado los desplazamientos al trabajo y sus tiempos, estando dominados en casi exclusividad por la carretera y, más específicamente, por el automóvil privado (Hoyos, 2006). También ha tenido lugar un aumento del tiempo de formación, tanto la previa como la que se realiza por las personas ocupadas, en muchos casos fuera total o parcialmente de la jornada de trabajo, trabajo de formación que debe ser considerado como socialmente necesario. De esta forma la pequeña reducción de la jornada laboral coexiste con un peso creciente del trabajo en la vida de las personas, por lo que está plenamente justificada la afirmación de que el tiempo de trabajo ha sido y sigue siendo un aspecto central de la relación salarial (Prieto y Ramos, 1999: 463). 


\section{Fin de la reducción de la jornada y cronificación del desempleo}

La 19. a Conferencia Internacional de Trabajo reunida en Ginebra el 4 de junio de 1935 constata que:

El desempleo se ha extendido tanto y se ha hecho tan persistente que en la actualidad millones de trabajadores, sin ser responsables de su situación, están en la miseria y sufren privaciones de las que legítimamente tienen derecho a ser aliviados", consideraba «que, para dar efecto a las resoluciones adoptadas por las reuniones decimoctava y decimonovena de la Conferencia Internacional del Trabajo, es indispensable desplegar un esfuerzo continuo a fin de reducir lo más posible las horas de trabajo en los empleos de todas clases (OIT, 2011)

Por esa razón, la Conferencia acordó el llamado Convenio de las 40 horas de 1935. En la época el Convenio no fue ratificado y con la Segunda Guerra Mundial el problema del empleo quedó postergado y solo después de la guerra fue introducida progresivamente en Europa la semana de 40 horas que condujo al pleno empleo. Ya para entonces, Russel (1932), afirma que "la técnica moderna ha hecho posible reducir enormemente la cantidad de trabajo requerida para asegurar lo imprescindible para la vida de todos».

Posteriormente y hasta la década de los setenta, el objetivo básico que inspiró la reducción de la jornada fue la conquista de descanso y tiempo libre de los trabajadores ${ }^{5}$. En las últimas décadas, esa tarea, sin desaparecer, cede la primacía al incremento del empleo: el papel fundamental que se le asigna es la contribución a la eliminación o al menos disminución del desempleo, pasando a ser una medida de política económica más que de política social (Alonso Olea, 1994: 71), papel que se acentúa ante la constatación del fracaso de las medidas tradicionales de fomento del empleo y la necesidad de "no poner en grave peligro las bases del sistema social» (Fernández Domínguez, 2000: 25). Se generaliza la idea de que el pleno empleo no puede resultar del crecimiento económico, y la reducción de la jornada debe ser un mecanismo de «reparto del empleo», mediante la reducción salarial correspondiente y/o la adopción de fórmulas flexibilizadoras en la organización del tiempo de trabajo (Ramírez Martínez, 1999: 3-4; Arriola y Vasapollo, 2003).

Tras un período de abandono de la RTT tras la Segunda Guerra Mundial, el movimiento sindical retomó este objetivo en Europa. Así, en 1978 el sindicato IG Metall reclamó en Alemania la implantación de la semana de las 35 horas y, en el mismo año, los sindicatos italianos publican el informe llamado Lavorare meno per lavorare tutti, cuyo título se convierte en el eslogan

\footnotetext{
5 Sobre el papel de las normas laborales en la fijación de topes máximos a la jornada, en especial para las categorías más explotadas de mujeres y niños véase Fita Ortega (1999: 16-17).
} 
más reconocido de la RTT. En 1979 la Confederación Europea de Sindicatos, en un congreso celebrado en Munich, recoge las reivindicaciones del $I G M e$ tall, sindicato, que tras la huelga más larga desde el fin de la Segunda Guerra Mundial, consigue en 1984 la semana laboral de 38,5 horas (Imaz Bengoetxea, 2006: 10; 2003).

La desaceleración del aumento de la productividad y el modelo de especialización sectorial en servicios de empleo estacional y bajo valor añadido (Recio, 2010), dio lugar antes de la última crisis a un "crecimiento más rico en empleos»: el umbral del crecimiento del PIB que desencadena la creación de empleo habría pasado en España del 2,5\% en $1975^{6}$ al 0,8\% de media del período 2001-2011 ${ }^{7}$. Este fenómeno de recuperación de la conexión entre empleo y crecimiento, que se había perdido en la década de los ochenta, es común a la mayor parte de los países europeos y se ha visto fortalecido por la reducción de los salarios, que hace menos rentable en algunos sectores productivos la introducción de innovaciones tecnológicas ahorradoras de mano de obra. Sin embargo, como resultado de una desaceleración del crecimiento, el empleo no ha alcanzado el ritmo de creación de la fase expansiva anterior, siendo un 2,32\% en el período 1994-1998, frente al 2,45\% del período 19861990 (Huete, 1999).

En el actual periodo de crisis del modelo de producción (Recio, 2010), distintos factores anulan esta capacidad motora del crecimiento sobre el empleo, al menos para la economía española, sometida a la fuerte presión de las políticas de ajuste fondomonetarista (Arrizabalo, 2014) y de devaluación salarial (Álvarez Peralta et al., 2013). En este proceso se va consolidando un «crecimiento sin empleo", a tenor - por ejemplo- de los datos de la EPA del primer trimestre de 2015, según la cuál se observa una destrucción de más de 114.000 empleos respecto al trimestre anterior (INE, 2015a) — principalmente fruto de la caída en el comercio y otros servicios tras la campaña navideña-. Esta caída y fuerte estacionalidad del empleo se conjuga con tasas de crecimiento del PIB de $0,9 \%$ en el mismo trimestre. Como explica Clavero (2015):

Si se cruzan las series históricas del PIB y de la EPA a partir de 2000, es posible observar que con una tasa así [de crecimiento del PIB] nunca antes se había perdido empleo. Todo lo contrario, siempre aumentó el número de puestos de trabajo, en una horquilla que va de los 72.000 de primer trimestre de 2007 a los 424.500 del segundo de 2005.

${ }^{6}$ Una de las razones que determinaba un escaso aumento del número de personas ocupadas, a pesar del intenso crecimiento del PIB, era la realización de un gran número de horas extraordinarias. Véase Huete (1999: 235).

7 Véase el estudio de Buendía Azorín y Sánchez de la Vega (2013). El estudio establece un porcentaje diferenciado, y mayor del $2,3 \%$, del crecimiento económico necesario para la reducción del desempleo. 
El crecimiento del empleo durante el periodo expansivo 1994-2007 estuvo basado en una coyuntura muy favorable debida a causas tales como una caída de las tasas de interés — que han alcanzado su nivel más bajo desde la Segunda Guerra Mundial_-, el auge del dólar que favoreció la actividad exportadora y, especialmente, el desarrollo de una burbuja inmobiliaria basada en el endeudamiento (López y Rodríguez, 2010). La temporalidad de la mayoría de las nuevas contrataciones y, sobre todo, la destrucción neta e irreversible del empleo indefinido y de la ocupación en la industria y la construcción — ver tablas 2 y 3 para Bizkaia, Gipuzkoa y Araba en anexo- son dos potentes vectores de la cronificación del paro que, de seguir por el actual camino, viene para quedarse en los próximos años. Así mismo, la reducción de las indemnizaciones de los despidos individuales y colectivos y, en general, el aumento de las facultades empresariales en la organización del trabajo que han realizado las últimas reformas laborales - especialmente la de 2012-, han dado lugar a un aumento del desempleo en cuantía superior a la que resultaría de la caída de la actividad económica. En efecto, como acredita Lago Peñas (2013), en España la relación entre el decrecimiento del PIB y la caída del empleo es mayor tras la reforma laboral de 2012 que en el período anterior.

Así pues, contrariamente a los argumentos que sostienen que el crecimiento económico acabará con el desempleo y proclaman la no necesidad de la RTT, creemos que no se puede dejar en exclusiva al crecimiento económico la tarea de eliminación del desempleo. España se mantiene, de forma persistente en los últimos años, en la tasa más elevada de desempleo en la Unión Europea (solo superado, en los últimos años, por Grecia). Esta cronificación del desempleo es hoy central en el debate económico, siendo sus magnitudes no recogidas correctamente en los indicadores al uso. Como puede observarse en las tablas 4 y 5 anexas, el aumento de la inactividad masculina y la fuerte activación de las mujeres entre 2008 y 2015, contrasta con la caída sostenida y no invertida —a la vista de la EPRA del tercer trimestre del 2015- del volumen de ocupados en los tres territorios históricos vascos observados (Eustat, 2015). La subestimación estadística del desempleo es fruto del aumento de la inactividad y de la no consideración del efecto de reducción de la población de 16 y más años — por razones demográficas o de migración laboral-.

Igualmente para Espańa, el INE (2014) nos muestra que, entre el primer trimestre del 2011 y el primero del 2014 según la EPA, se da un aumento de los «inactivos desanimados» hasta más de 483.000 (85.000 más en este periodo), al tiempo que la población de entre 25-34 años se reduce en más de un millón, sin poder descomponerse el efecto demográfico y el de exilio económico. Éste último es estimado por Juventud Sin Futuro en unos 220.000 menores de 35 años en los primeros cuatro años de crisis (Muñoz, 2013). De igual manera para Hego Euskalerria, Gaindegia (2013) nos recuerda que 22.400 jóvenes de 18 a 34 años se fueron a Espańa o a otros países, contando sólo las partidas de 2012. 
Por tanto, en este contexto de cronificación del desempleo — cada vez menos generador de derechos contributivos ${ }^{8}$ - , una de las variables principales que determina las condiciones laborales en las economías salariales de servicios (Albarracín, 2003; Zubiri, 2014) es el tiempo de trabajo. Trabajar todo el mundo menos daría posibilidades para, tal vez, acabar generalizando el acceso a una ocupación remunerada a toda persona que manifestara su voluntad de acceder a ella. El tiempo de trabajo y la jornada laboral determinan a cuántos empleos dará lugar un cierto volumen de horas de trabajo, y es ese primer factor el que no está manteniendo una evolución que permita aprovechar las potencialidades del crecimiento para la creación de empleo9. Por una parte, porque la variable tiempo de trabajo, que interviene en el volumen del empleo, ha sufrido un cambio en su tendencia histórica: la RTT se ha estancado durante los últimos años, durante algunos de los cuales incluso se han llegado a producir incrementos, como se acredita en las estadísticas de los convenios colectivos. Hay que añadir, además, que los especialistas señalan que no existen fuentes estadísticas que proporcionen informaciones ajustadas a la realidad del tiempo de trabajo efectivamente realizado, siendo la razón fundamental que los informantes, cuando responden a encuestas, tienden a ofrecer respuestas que se adecúan a la normativa pública — sobre jornada en este caso- (Prieto y Ramos, 1999: 471) ${ }^{10}$.

Por otra parte, porque tal como viene señalando el ecologismo, la economía no puede seguir obviando por más tiempo los límites físicos del planeta, lo que exige un nuevo modelo productivo con pautas de crecimiento de la ocupación laboral que se dirijan hacia sectores ${ }^{11}$ que minimicen los impactos irrever-

8 ELA (2015a) constata que "en el mes de julio de 2015 el 55,3\% de las personas en desempleo en Hego Euskal Herria no percibia prestación alguna, el 26,1\% tenia una prestación contributiva y el 18,6\% percibia subsidios o la Renta Activa de Inserción; asi, son ya 109.244 las personas [paradas inscritas] que no perciben ninguna renta. El escaso nivel de cobertura a las situaciones desempleo es una de las causas principales para explicar el descenso de la población activa». En julio del 2013 eran todavía 45,6\%, lo que muestra que "entre 2013 y 2015 dejaron de percibir prestaciones contributivas 24.995 personas y 8.413 personas menos perciben el subsidio o la Renta Activa de Inserción». Esto origina la paradójica evolución del gasto mensual en cobertura por desempleo — prestaciones y subsidios_ de 136 millones en julio del 2013 a 88 millones en el mismo mes de 2015.

9 Para un estudio de la actividad, el empleo y la jornada laboral en Gipuzkoa ver las tablas en anexo y, sobre todo, Castrillón et al. (2016a; 2016b; 2016c).

10 Véase el estudio sobre los acuerdos colectivos en relación con el tiempo de trabajo de Eurofound (2014). Conforme a este estudio la jornada media semanal acordada en la negociación colectiva era en el 2013 de 38,1 horas semanales, igual que en el 2012. La jornada anual era de $1.711,8$ horas en la UE 28, de $1.678,5$ en la UE 15 y de $1.715,8$ en España.

11 Según Gadrey, Marcellesi et al. (2013) los más importantes son:

- Fabricación, instalación y mantenimiento de redes de energías renovables.

- Alojamiento social y rehabilitación integral de inmuebles (confort climático y uso de energía eficientes, accesibilidad sin barreras, auto-abastecimientos...).

- Renovación del transporte y movilidad sostenible.

- Separación en origen y reciclaje integral de residuos.

- Producción agrícola ecológica y/o local. 
sibles al medio ambiente. En buena medida, estos sectores se corresponden con necesidades sociales y ambientales insatisfechas ${ }^{12}$. Las administraciones públicas tienen una responsabilidad esencial en los servicios públicos, sociales y medioambientales y deben jugar un papel tractor en esta transformación a todas luces inevitable, mejor más temprano y de forma, por tanto, más ordenada que tarde y por imperativos exógenos (Fernández Durán et al., 2015). En sentido inverso, cuando se han producido privatizaciones en la provisión de estos servicios, ha tenido lugar una disminución del empleo y pérdida de su calidad y viceversa que ha sido, en parte, ejecutada mediante la modalidad del tiempo parcial forzado, aspecto del que nos ocupamos en el siguiente apartado.

\section{Tiempo parcial forzado, en vez de reducción general de la jornada}

A falta de un cambio de orientación en este sentido, Riechmann y Recio (1997: 36-37) apuntan "la imposibilidad de que una economia capitalista genere pleno empleo de forma sistemática", fruto de "los problemas de coordinación de una economía descentralizada que se basa en los estados de humor de una minoría rentista» y de "politicas diseñadas para mantener elevado el nivel de desempleo». Así, en la actualidad se está poniendo en práctica en todo el sur de Europa una reducción del tiempo de trabajo, pero de una forma profundamente discriminatoria, a través del paro masivo, el fomento de la inactividad forzosa y del trabajo a tiempo parcial. En los últimos años, está teniendo lugar un enorme crecimiento de este tipo de contratación.

En España el tiempo parcial alcanzaba al 16,05\% de las personas asalariadas en el último cuatrimestre de 2014, eso sí con un importante sesgo según el sexo: el 73,03\% de los contratos a tiempo parcial correspondía a las mujeres. En términos absolutos, entre el tercer trimestre de 2008 y el tercer trimestre de 2014 aumenta el número de personas empleadas a tiempo parcial en 325.700, un 14,2\% y el número de horas trabajadas a tiempo parcial en 4.685 .600 (un 12,9\%). De 2013 a 2014, mientras que aumentó la contratación a tiempo completo en 119.600 hombres, tan sólo lo hizo en 34.100 mujeres. En relación con la con-

— Sanidad y enseñanza públicas de calidad.

- Atención a las personas con necesidades especiales de cuidado.

- Protección y aprovechamiento sostenible de los entornos naturales.

12 La mayoría de estos sectores son de muy alta intensidad de empleo y compensarían sobradamente las pérdidas de puestos de trabajo que se producirían por la necesaria anulación o reducción de la energía fósil y nuclear, de la industria armamentista y otras similares. Así mismo, para el caso vasco, una apuesta por estos sectores, con los necesarios estímulos públicos tractores, podría suponer la creación de 45000 nuevos «eco-empleos» (Marcellesi, 2012), sin necesidad de grandes inversiones de capital por unidad de producto y con sustanciales retornos no sólo sociales y ecológicos, sino también fiscales y de multiplicador extensivo al comercio y otros ámbitos de la economía. 
tratación indefinida a tiempo completo, en el caso de las mujeres ha experimentado un descenso, pasando a ser de un $42,72 \%$ en 2013 a un $42,57 \%$ en 2014, mientras que la masculina se ha incrementado de un $57,27 \%$ a un $57,42 \%$. Estos datos éstos confirman la tendencia hacia una mayor precarización del empleo femenino. Igualmente, el CES (2015) nos muestra para la CAPV que el empleo a jornada completa - temporal o indefinido- recula en casi 52.000 efectivos de final del 2011 a final del 2014, mientras que el empleo a tiempo parcial aumenta en casi 9.000 efectivos durante el mismo periodo, pasando así del $20 \%$ al 22,7\% de las personas afiliadas al Régimen General de la Seguridad Social. Por último, en relación a los nuevos contratos, casi el 39\% de todos los firmados en la CAPV entre enero y mayo del 2015 fueron a tiempo parcial (ELA, 2015b).

Según la Encuesta Trimestral de Coste Laboral (ECTL) del primer trimestre de 2015 , la jornada semanal media pactada, considerando conjuntamente a trabajadores a tiempo completo y tiempo parcial es de 149,0 horas mensuales en España y 147,2 en la CAPV (INE, 2015b). Según la misma ECTL la jornada efectiva del tiempo parcial es poco más de la mitad de la correspondiente al tiempo completo.

Tabla 1

Horas por trabajador y mes (jornada pactada). España y CAPV

\begin{tabular}{lccc}
\hline & Total Asalariadas & Tiempo completo & Tiempo parcial \\
\hline España & 149,0 & 168,5 & 84,9 \\
CAPV & 147,2 & 164,7 & 78,7 \\
\hline
\end{tabular}

Fuente: ECTL del primer trimestre de 2015 (INE, 2015b).

La contratación a tiempo parcial tiene unas características muy negativas $y$, junto a los contratos temporales, se considera que constituye un supuesto de baja calidad del empleo. Este aspecto se acentúa por la creciente involuntariedad de los mismos, ya que en una elevada proporción el contrato a tiempo parcial se acepta forzosamente en defecto de uno a jornada completa. Así, en la UE ha tenido lugar un importante crecimiento de la involuntariedad del tiempo parcial y del trabajo temporal —especialmente en Irlanda, España (que siempre se ha caracterizado por este rasgo del tiempo parcial ${ }^{13}$ ), Chipre, Grecia e Italia- y alcanza el 29,6\% en el 2013, con un porcentaje superior al 70\% en Grecia y ma-

13 Según un informe del CES (1996, p. 26), España tenía en 1995 el índice de voluntariedad más bajo de la UE: sólo el 3,9\% de los hombres y el 3,6\% de las mujeres declaraba trabajar a tiempo parcial por no haber querido un trabajo a tiempo completo. 
yores del 60\% en Italia y España, tal como se acredita en el siguiente gráfico (ETUI, 2015: 30):

Figura 1

Empleo tiempo parcial involuntario, en\% del total, en 2008 y 2013

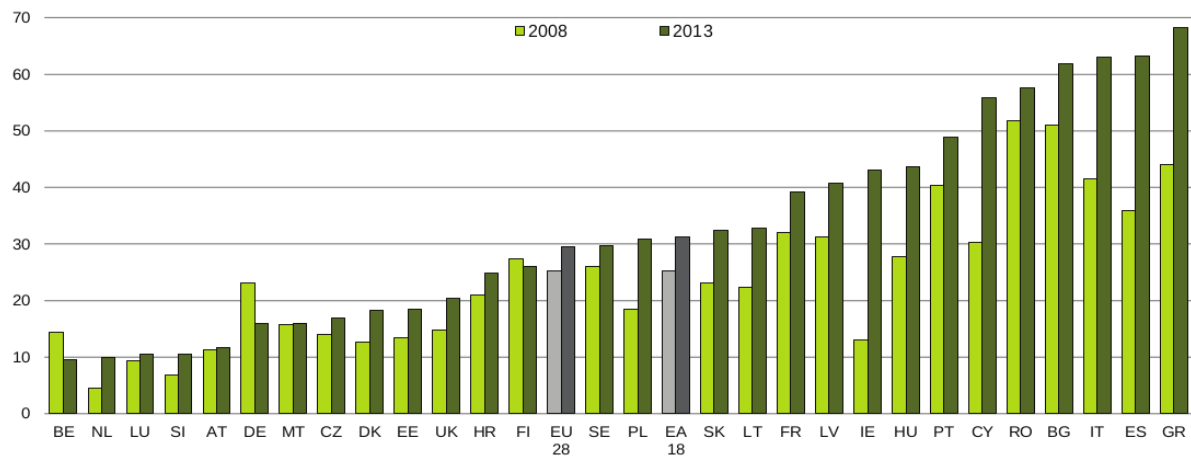

Source: Eurostat [lfsa_eppgai]. Notes: UK data for 2009 (not 2008).

Igualmente, UGT (2015: 9) muestra que según la EPA la proporción de involuntariedad en el tiempo parcial en España era de menos del 35\% hasta el 2008, subiendo desde entonces y situándose en casi el $65 \%$ en la segunda mitad de 2014. Además, la aceptación voluntaria por las mujeres de los contratos a tiempo parcial, cuando exista (por ej. en algunos casos en el sector público), está enmarcada en la división sexista del trabajo, con el consiguiente "peligro de legitimación de un nuevo modelo: los hombres trabajando a jornada completa en el mercado y las mujeres repartiendo su tiempo entre el trabajo doméstico y un trabajo asalariado a jornada parcial» (Carrasco, 1995: 35) ${ }^{14}$, así como por las limitaciones y no gratuidad de las estructuras de acogida de los niños/as —haurreskolak-. Les supone además, las evidentes desventajas que implican estos contratos en cuanto a oportunidades de promoción, menor utilización de sus aptitudes profesionales, protección social reducida, etc. En la doble concepción de que, para el trabajador individual «el tiempo parcial y el tiempo flexible son tanto promesas de libertad como amenazas de subordinación incrementada» (AA.VV., 1998: 97), es la segunda opción la que se impone en la práctica.

14 Torns (2007: 273-4), a las razones expuestas que explican la más alta involuntariedad del trabajo a tiempo parcial de las mujeres en Espańa, en relación con otros países europeos, de la actual precariedad laboral de las mujeres y de las peores condiciones laborales y salariales de los trabajos a tiempo parcial, añade la protesta frente al modelo male breadwiner, en el que las mujeres asumen la parte del trabajo doméstico y de cuidados. Para profundizar en esta cuestión ver Carrasco et al. (2011). 
A la involuntariedad del tiempo parcial se une la flexibilidad de los horarios —que ahora se incrementa aún más con la nueva regulación de las horas complementarias ${ }^{15}$ - y va unido, de forma muy mayoritaria, a los bajos salarios. En efecto, según la Encuesta Trimestral de Coste Laboral del primer trimestre de 2015 (INE, 2015b), la diferencia salarial entre los trabajadores a tiempo completo y parcial es de 4,62 euros por hora (14,29 euros/hora de media para tiempo completo frente al 9,67 para tiempo parcial), lo cual da buena muestra de las pautas de intensificación del tiempo parcial en sectores feminizados y peor remunerados.

\section{Algunas cuestiones, dificultades y ventajas de la puesta en práctica de la RTT}

Todo lo expuesto —el relativo estancamiento de la reducción de las jornadas, la cronificación del paro y la imposición creciente del empleo a tiempo parcial-, es sólo una muestra de las tendencias que una política de RTT podría contrarrestar en donde fuera aplicada ${ }^{16}$. La estrategia de RTT se enmarca en la llamada «flexibilidad ofensiva», que requiere un modelo intervencionista de relaciones laborales (Monereo Pérez y Medina Castillo, 1999:35), que en nuestro caso se basa en la esencia del Estado Social y Democrático de Derecho (art. 1 de la Constitución Española, CE), que obliga a los poderes públicos a remover los obstáculos que dificulten que la libertad e igualdad sean reales y efectivas (art. 9.2, CE), de forma que se garantice el derecho al trabajo y empleo para todos (arts. 35.1 y $40.1 \mathrm{CE}$ ) y se cumpla el mandato que ordena a los poderes públicos fomentar «...una politica que garantice (...) el descanso necesario, mediante la limitación de la jornada laboral...” (artículo 40-2, CE) ${ }^{17}$. Su eventual carácter redistributivo de la riqueza tiene asimismo basamento constitucional: «toda la riqueza del pais, en sus distintas formas y sea cual fuere su titularidad está subordinada al interés general» (art. $128 \mathrm{CE})$.

15 La regulación del tiempo parcial se examina también en Bengoetxea (2016).

16 A lo largo de todo el informe de restitución de la investigación financiada por el Departamento de Innovación de la Diputación Foral de Gipuzkoa, hemos aportado elementos de diagnóstico —Castrillón et al. (2016a; 2016b, 2016c) - y unas primeras proyecciones y cálculos para apuntar hacía la viabilidad competencial, fiscal y normativa de la Diputación para llevar a cabo medidas en el campo de la RTT (Castrillón et al., 2016d), buscando también una coordinación programática con el resto de herrialdes vascos, incluido el navarro cuyo Acuerdo programático para el Gobierno de $\mathrm{Na}$ varra recoge la voluntad de profundizar en la vía de la RTT-Bloque programático n. ${ }^{\circ}$ 3: ECONOMÍA, EMPLEO Y FISCALIDAD, INDUSTRIA, COMERCIO, TURISMO Y RELACIONES LABORALES; Sección: Relaciones laborales; Puntos 7-9 (Geroa Bai et al., 2015: 43).

17 Para el Tribunal Constitucional, «en su dimensión colectiva el derecho al trabajo implica además un mandato a los poderes públicos para que lleven a cabo una politica de pleno empleo, pues en otro caso el ejercicio del derecho al trabajo para una parte de la población lleva consigo la negación de ese mismo derecho para otra parte de la misma» (STC 22/1981, de 2 de julio). 
La reducción del tiempo de trabajo está estrechamente relacionada con la distribución de los ingresos. La implantación de una RTT sin reducción de salarios debe permitir la recuperación de una parte de lo producido, que en las últimas décadas ha ido a los beneficios empresariales y en detrimento de los salarios. La recuperación de las pérdidas salariales, que en los ańos de la crisis ha dado lugar incluso a una pérdida del poder adquisitivo de los salarios, está plenamente justificada, en especial en algunos sectores productivos y empresas ${ }^{18}$. Sin embargo consideramos que, de priorizar entre ambas medidas —reducción de sueldo o de jornada - , hay que hacerlo, en general, a favor de la RTT, pero asegurando la progresión salarial de los trabajadores más precarizados y de los trabajadores pobres. En la opción entre incremento salarial y RTT como elementos para la mejora del empleo, desde el ángulo simbólico y político la legitimación social es incomparable en un caso y en otro, a favor de la primera vía a pesar de estar hoy bloqueada por la coyuntura política y económica.

El mantenimiento de los salarios en la propuesta que formulamos incluye el efecto de arrastre de la RTT sobre otros países europeos (Coutrot, 1997: 53). Esta cuestión del marco europeo es clave para soslayar el problema de la competitividad de las empresas que se suele argüir como obstáculo para la RTT ${ }^{19}$. El grueso de los intercambios comerciales de la CAPV se realiza en el interior de la Unión Europea. El poderío económico de la UE y su grado de independencia en relación con el exterior ${ }^{20}$, permite extender una RTT progresista y solidaria, sin que las empresas europeas tengan que sufrir graves problemas de competitividad. Los efectos positivos de la RTT, en términos de creación de empleo, aumentarían con la generalización de la medida a otros países europeos. En efecto, Navarro (1999) mostraba hace ya un tiempo que durante el proceso de globalización mundial e integración europea, varios Estados han realizado políticas expansivas de tipo redistributivo — similares a una RTT con mantenimiento de salarios-, a pesar de que en algunos casos, sus economías estaban especialmente abiertas al exterior. Este y otros estudios muestran, asimismo, que los retrocesos

18 Según el diagnóstico sobre el empleo en Gipuzkoa realizado por Castrillón et al. (2016a; 2016b; 2016c), la evolución positiva de las productividades en mayor medida que las remuneraciones - y, por tanto, la tendencia estable y a la baja de los costes laborales unitarios-, permite concluir la necesidad de compensación global a las personas asalariadas, siendo además la evolución anual y semanal de jornadas a tiempo completo poco decreciente en las últimas décadas

19 El espacio europeo permite eludir la disminución del margen macroeconómico de los Estados/Nación provocado por la globalización. Véase Monereo Pérez et al. (2000: 78).

20 Las exportaciones de bienes y servicios de la zona euro representaban en 2013 el $45 \%$ del PIB y sus importaciones el 42\% (Eurostat STAT/14/110, 17-7-2014). Pero esos datos son engańosos ya que incluyen las transacciones en el interior de la Unión Europea. Descontadas las mismas, la apertura sería mucho menor. Así, mientras que el cociente de la suma de exportaciones e importaciones en relación con el PIB era del 58,9\% en el año 2000, si se descuentan las transacciones internas el porcentaje cae al 23,9\%. Véase sobre esta cuestión de la apertura económica y los bloques comerciales, Schvarzer (2004). 
en esas políticas se han debido, no al proceso de globalización, sino a decisiones políticas específicas, tomadas en distintos niveles nacionales e internacionales, en beneficio del capital financiero (Lordon, 2008; Álvarez Peralta et al., 2013).

En este sentido es muy sugerente la propuesta de ATTAC Alemania de poner en práctica la semana de 30 horas (ATTAC Alemania, 2015), tal y cómo reivindica la CNT-AIT desde finales de los 90 en los casos español y vasco (Fuster y Rodríguez., 2013). La eficacia de la RTT en la creación de empleo y, también, en la oposición a la intensificación del trabajo, exige un control de los trabajadores/as sobre la realidad de las creaciones de empleo. Esto puede aplicarse, por ejemplo, en la elaboración de planes de nuevas contrataciones que tengan en cuenta las necesidades efectivas de personal, lo penoso de los horarios flexibles o atípicos o de la necesidad de eliminar los trabajos precarios, temporales y a tiempo parcial. La experiencia de las Leyes Aubry en Francia (De la Fuente y Zubiri., 2016b) muestra que las medidas flexibilizadoras que acompañaron a la puesta en práctica de la reducción de la jornada, no solo redujeron su impacto en la creación de empleo sino que debilitaron progresivamente el apoyo a la misma por parte de la población trabajadora, que en muchos casos consideraba que se veía abocada a «hacer lo mismo» que antes, pero en menos tiempo.

La RTT colectiva y generalizada constituye una alternativa al reparto del trabajo a través del desarrollo del tiempo parcial ${ }^{21}$, como hemos visto mayoritariamente femenino e involuntario. Si la RTT va acompañada del mantenimiento en cotas importantes del trabajo a tiempo parcial existe un grave riesgo de que se mantengan las desigualdades entre los trabajadores a tiempo completo y los de tiempo parcial. Así mismo, la experiencia nos muestra que estas modalidades de empleo atípico o precario refuerza la reproducción de los roles imperantes en la división sexual del trabajo, lo que relega a las mujeres a las esferas más invisibles y desvalorizadas de la actividad social, tanto remunerada como no remunerada.

El instrumento característico para asegurar la generalización de la RTT es la ley (Bengoetxea, 2016). En España, la competencia de la legislación laboral, que incluye la regulación de la jornada laboral, está atribuida con carácter exclusivo al Estado (art. 149.1.7. a de la CE). Otra vía posible para acordar medidas de RTT generalizadas es el convenio colectivo, ya que el art. 81.2 del Estatuto de los Trabajadores (ET), establece que a través de estos, «en su ámbito correspondiente», «los empresarios y trabajadores regulan las condiciones de trabajo y productividad», entre las cuales está obviamente incluida la jornada (también el art. 85.1). Conforme a los apartados 2 y 3 del art. 83 del ET, las organizaciones de trabajadores y empresarios "de carácter estatal o de Comunidad Autónoma», pueden establecer «mediante acuerdos interprofesionales» acuerdos «sobre materias concretas», por

${ }^{21}$ El desarrollo del empleo a tiempo parcial es central en el modelo holandés de reducción del tiempo de trabajo (Jean, 2000; Visser, 2000). 
ejemplo la jornada, acuerdos que «tendrán el tratamiento de esta Ley para los convenios colectivos». Es obvio que la suscripción de un acuerdo de este tipo exigiría una presión sindical y social muy elevada, así como la colocación de la RTT en la agenda de los sindicatos, como medida más eficaz en tiempos de crisis tanto para favorecer una mayor distribución a los trabajadores de los excedentes económicos producidos como para, así mismo, incentivar una mayor libertad en los usos de los tiempos de vida más allá del trabajo asalariado.

Husson (2015) muestra — para una serie larga del caso francés-, que en la época de fuertes incrementos de la productividad no se generaba paro y que el desempleo de masas que se gesta a partir de la década de 1980 es sobre todo fruto de "una insuficiente reducción del tiempo de trabajo». Como exponen Husson y Treillet (2014) el mayor problema para la asunción por el movimiento obrero y sindical de este objetivo de la RTT está relacionado con el balance negativo que interioriza una parte significativa de la clase asalariada de sus modalidades de aplicación (aumento de la flexibilidad y carencia de nuevas contrataciones). Alonso y Pérez (1996: 73) recuerdan que, además de diferenciado por sectores, el posible aumento del empleo fruto de una RTT se producirá siempre con cierto retardo y en determinadas condiciones, pudiendo ser esta "remuneración en especie de las mejoras de productividad» generadora de cambios productivos y organizativos que anulen el efecto de la creación de empleo. Así mismo, las consideraciones de Riechmann y Recio (1997: 57-58) sobre las reducciones salariales que se proponen en algunas acepciones menos redistributivas de la RTT —o, como dicen ellos, de «reparto del paro»—, dan la impresión de que una RTT digna de su nombre requeriría de una relación de fuerzas sociales más favorable a la clase trabajadora que la actual.

A lo anterior se agregan los efectos de cierta obsesión ideológica, también en el interior de una parte de la izquierda, conforme al cual es necesario escoger entre el empleo y los salarios, dando por sentado que la distribución actual del valor agregado debe permanecer inalterable. Esta conjunción de opciones de ocupación y condiciones de distribución es fruto del cuadro macroeconómica global y nacional, así como de aspectos regulatorios, productivos y fiscales. ELA (2015b) calcula que "en 2013 se destinó el 47,7\% del PIB del Estado español a las rentas de trabajo", mientras "en la CAPV se destina un $49 \%$ del PIB a las rentas de trabajo (y el $51 \%$ a las rentas de capital)». En el último caso las rentas del trabajo eran el $51,7 \%$ del PIB en 2009. Esto significa que «lo destinado a las rentas de trabajo está rozando mínimos históricos mientras lo destinado las rentas de capital ha marcado el máximo de los últimos 14 años».

Más allá de la importancia de revertir también esta devaluación salarial, consideramos estratégico en los tiempos presentes abordar la eficacia histórica y la actualidad de las políticas de RTT, para lo cual presentamos este monográfico completo en Lan Harremanak, con el fruto de las indagaciones de nuestro 
grupo de investigación en la materia. En lugar de jornadas a tiempo completo de elevada duración, en muchos casos ampliadas con horas extraordinarias, mayoritariamente realizadas por los hombres y, por otra parte, jornadas a tiempo parcial que corresponden de forma muy mayoritaria a las mujeres, la reducción generalizada del tiempo de trabajo, con el establecimiento de jornadas más cortas, permite un reparto más equitativo del trabajo doméstico y de cuidado. Sin embargo, aunque una RTT sustancial, equitativa y redistributiva es una condición indispensable para el cuestionamiento de los papeles sociales y de la división sexuada de las tareas domésticas y de cuidado (Carrasco et al., 2011), debe ir acompañada de una educación no sexista y el desarrollo de los servicios públicos de cuidado y acogida (Husson y Treillet, 2014). Por otra parte, se debe optar por las modalidades de reducción que faciliten esa tarea, priorizando la reducción de la jornada diaria en lugar de más días libres.

\section{Conclusiones}

En este artículo hemos señalado algunos elementos sobre el significado histórico y la actualidad de las políticas Reducción del Tiempo de Trabajo (RTT). En el primer apartado de introducción hemos contextualizado el tema desde los inicios del movimiento obrero por la RTT, objetivo siempre en primera línea de las reivindicaciones de los trabajadores para un mejor reparto de los excedentes productivos y la consolidación de tiempos libres de vida. Siendo las huelgas y el movimiento internacional por las 8 horas diarias el de mayor relevancia histórica, se observan desde 1919 hasta hoy leves reducciones de jornada en la mayoría de países europeos. En esta tendencia al estancamiento de las políticas de RTT, se dan por contra varios factores que muestran una cierta reducción de la jornada semanal de trabajo en la Unión Europea: la reducción de la población que realiza jornadas largas; el aumento de la población con jornadas cortas; los acuerdos colectivos en algunos países; y las reducciones más bien ocasionadas por la crisis desde 2008.

Acto seguido, hemos puesto nuestra atención en dos cuestiones centrales en el estudio del tiempo de trabajo: la realización de horas extraordinarias y la contrapartida flexibilizadora de la mayoría de políticas de RTT. En el primer plano cabe destacar el aumento de las horas extraordinarias no pagadas y las caídas no registradas del salario hora que implican, así como, en el caso de las pagadas, su creciente desfiscalización y regulación desreguladora ${ }^{22}$. En el segundo caso pode-

22 Recientemente la patronal CEOE ha reclamado una ampliación sustancial de la posibilidad legal de las horas extraordinarias, mediante la introducción en los convenios colectivos del «aumento de las horas extraordinarias por encima de las 80 horas al ańo establecidas en el Estatuto de los Trabajadores», hasta alcanzar la jornada laboral máxima anual de 1.826 horas y 27 minutos, compután- 
mos encontrar toda una gama de modalidades de RTT que van acompañados de una creciente irregularidad de las jornadas, de flexibilización de horarios y de creciente conflicto entre los espacios de trabajo productivo para el mercado y los de cuidado y reproducción de la vida. Otros factores como el aumento de los desplazamientos, y la fuerte sobrecarga que implica para mucha gente en relación a los tiempos dedicados al trabajo, se suman a otras mutaciones sociales omnipresentes como la generalización de las tecnologías de comunicación en nuestras vidas.

En el segundo apartado nos hemos entregado a un estudio más detallado de la reducción de la jornada más drástica e incidente hoy en día, la de la cronificación de partes crecientes de la población trabajadora en un desempleo involuntario que no tiene sino múltiples consecuencias nefastas. Si hubo una época en la que se consideraba la permanente RTT una cuestión de primer orden macroeconómico para los trabajadores, en las últimas décadas la consolidación de un paro estructural de masas da pie a hablar de la RTT como medida para crear empleo. Lavorare meno per lavorare tutti sigue siendo una consigna central en el imaginario global de los avances de la clase trabajadora, a pesar del estancamiento encontrado en los tiempos presentes tanto en la creación de empleo como en la RTT.

Para el caso español, diversos indicadores nos muestras un crecimiento cada vez menos rico e intenso en generación de nuevas ocupaciones remuneradas. Esto da lugar a tasas de crecimiento del PIB que cada vez son menos tractoras sobre el empleo, los salarios directos y las cotizaciones sociales, menos todavía si se tiene en cuenta la estacionalidad y la precarización global que la nueva especialización de servicios de las economías supone para las clases trabajadoras, cada vez más de rostro femenino. Este proceso que lleva incubándose las últimas décadas — sobre todo desde la salida de la crisis de 1993-94_, ha dado paso en

dose sólo a partir de ese máximo el límite de las 80 horas extraordinarias. Ello supondría "eliminar la cotización de las horas extraordinarias» a la Seguridad Social, lo que supondría una reducción del coste para las empresas que oscilaría entre el 12\% y el 23,6\%, en función del tipo de horas extraordinarias. Asimismo pretende reducir el control sobre la realidad del horario extraordinario, mediante la supresión de la exigencia legal de llevanza de un libro registro en el que constan las horas extraordinarias realizadas. Por último, pero no menos importante, la CEOE reclama la supresión de la actual prohibición de realizar horas extraordinarias en el trabajo nocturno - lo que afectaría gravemente a la seguridad y salud de los trabajadores - y en los contratos a tiempo parcial. Se advierte que en este tipo de contratos existe la posibilidad de realizar las llamadas horas suplementarias, por lo que se introdujera la posibilidad de horario extraordinario ello supondría una flexibilidad extrema de disposición de la jornada por parte de los empleadores, con una garantía muy baja de salario para los trabajadores.

Estas propuestas se se recogen en el borrador de un documento de la Comisión de Diálogo Social de la CEOE sobre Propuestas laborales para el crecimiento de la economía española, según información que se recoge en Valverde (2016). Para una revisión del marco jurídico sobre la reducción del tiempo de trabajo en nuestro entorno ver Bengoetxea (2016). 
los últimos años a una cronificación del desempleo, que se plasma en una caída sostenida del volumen de ocupados hombres en términos inter-anuales ${ }^{23}$ y una creciente incidencia del empleo y el paro femenino. Esto implica aumentos de las personas inactivas que, desanimadas y cada vez menos generadoras de derechos, se introducen en un itinerario que tiene dos puertas principales: el exilio económico o el atrapamiento en la precariedad sociolaboral como horizonte vital. Hemos dedicado parte de este apartado a discutir algunos aspectos sobre el tiempo de trabajo y también hemos apuntado vías de ocupación laboral viables y necesarias en una transición socio-ecológica de nuestras economías.

En el tercer apartado hemos constatado que la contraparte de este desempleo - que afecta a jóvenes de forma generalizada - es la creciente activación y sub-empleo de las mujeres. Hemos entrado más en detalle en la otra vía de RTT forzada y regresiva en términos distributivos que está ganando incidencia en los últimos ańos, el empleo a tiempo parcial. De incidencia creciente, mayoría femenina y salarios sustancialmente más bajos, hemos podido constatar también el espectacular aumento de su carácter forzoso en toda Europa. Siendo aquí España uno de los países en los que esta evolución ha sido más notoria. Hemos hecho algunas apreciaciones sobre esta proliferación del empleo a tiempo parcial, claramente orientado en toda Europa a las mujeres, remarcando el rol de refuerzo del reparto sexista del trabajo que implica. Así mismo las horas extras son, en España, crecientemente no pagadas y concentradas en los hombres, en un contexto de infra-utilización general de las fuerzas productivas en las economías de nuestro entorno.

Por último, una cuarta y última sección ha planteado algunas cuestiones, dificultades y ventajas de la puesta en práctica de políticas ofensivas de RTT, a favor de un mejor reparto global de excedentes y de tareas productivas y reproductivas en nuestras sociedades. Con este cometido, hemos empezado enmarcando la lucha por la RTT en la llamada «flexibilidad ofensiva», que además de su idoneidad en la consecución de los intereses de la mayoría asalariada, se fundamenta también en su cobertura legal del más alto rango. Acto seguido, se ha considerado que la RTT es hoy día una vía más adecuada que la reivindicación de mayores salarios. Esto debe ser compatible con un aumento sustancial de los salarios más bajos y, en particular del salario mínimo interprofesional, ya que puede dirimir a favor de las personas trabajadoras el conflicto distributivo actual, sin por ello poner en riesgo la competitividad de las economías en las que

23 En el diagnóstico hemos vuelto a identificar los focos de atención de nuestras propuestas, que es resolver de forma consistente el aumento masivo del desempleo, tras la destrucción de $60.000 \mathrm{em}$ pleos industriales y 32.000 en la construcción en la CAPV, sólo entre 2008 y 2014 - Tabla 2 en anexo-. Hemos visto que el paro de larga duración aumenta si se tiene en cuenta la inactivación y podemos ver también una creciente parte de los desempleados activos que no reciben prestaciones ni subsidios. 
esta política fuera aplicada. Además de poner en alza algunas experiencias sindicales que ahondan en la necesidad de que la reducción propugnada sea sustancial, dejando la jornada semanal en unas 30 horas, se ha concluido con una consideración de la RTT como una forma de remuneración en especie del todo viable tras ańos de perjuicio para la clase asalariada de los incrementos de productividad acumulados en décadas por las economías capitalistas. Por último se ha remarcado la importancia de aplicar políticas de RT'T de cara a un reparto equitativo de los trabajos domésticos y de cuidados.

Todo lo expuesto nos ha permitido concluir que la RTT colectiva y generalizada constituye una alternativa al reparto del trabajo a través del paro cronificado y el desarrollo del tiempo parcial, ambos con un fuerte sesgo según el sexo. Múltiples estudios dicen que uno de los problemas de las economías europeas es la no canalización de partes mayores de excedentes a salarios, siendo una medida más sencilla que el aumento de salarios la RTT sustancial, equitativa y redistributiva que propugnamos, condición además indispensable para el urgente reparto entre hombres y mujeres de los trabajos de cuidados. Hemos dado especial importancia a clarificar en toda estrategia de RTT la cuestión de la compensación salarial, o quién financia el aumento del coste laboral. Cómo se desarrolla mejor en Castrillón et al. (2016d), nuestra posición es favorecedora de una RTT sustancial, redistributiva - financiada por el capital y los salarios elevados y muy elevados 24 _ y equitativa — que sea para la mayoría y no genere dualidad entre unos trabajando igual y otras bajando a jornadas reducidas o muy reducidas- Esperemos que esta línea de sentido común se imponga próximamente en la ineludible transformación del modelo laboral y productivo de las economías europeas.

\section{Bibliografía}

AA.VV. (2007) (Prieto, Carlos ed.): Trabajo, género y tiempo social, Madrid, Hacer-Editorial Complutense

AA.VV. (Alain Supiot, dir.)(1998): Transformation du travail et devenir du droit du travail en Europe. Conclusions du rapport, Luxemburgo, Comisión Europea.

Albarracín Sánchez, Daniel (2003): De la utopia postindustrial a la crisis de las sociedades salariales de servicios: En torno al ciclo del capital y la identidad de clase de los trabajadores españoles en el capitalismo tardio, Tesis Doctoral dirigida por Luis Enrique Alonso Benito, Madrid, UCM, 638 p.

Alonso, Luis Enrique y PÉrez Ortiz, Lourdes (1996): ¿Trabajo para todos?: Un debate necesario, Madrid, Editorial Ediciones Encuentro, 140 p.

24 No hay que olvidar que los salarios muy elevados en muchos casos corresponden a directivos que son también accionistas, siendo a nuestro entender conveniente en los tiempos presentes fijar límites en relación con los salarios más bajos, por ejemplo que los más altos no puedan sobrepasar 6 veces los salarios más bajos dentro de una empresa o sus subcontratas orgánicas. 
Alonso Olea, Manuel (1994): Introducción al derecho del Trabajo, 5. ${ }^{a}$ ed., Civitas, Madrid, 1994.

Álvarez Peralta, Nacho, Luengo, Fernando y Uxó, Jorge (2013): Fracturas y crisis en Europa, Madrid, Ed. Clave Intelectual, 342 p.

Arriola, Joaquín y Vasapollo, Luciano (2003): Flexibles y Precarios: La opresión del trabajo en el nuevo capitalismo europeo, Barcelona, El Viejo Topo.

Arrizabalo Montoro, Xabier (2014): Capitalismo y Economía Mundial: Bases teóricas y análisis empírico para la comprensión de los problemas económicos del siglo XXI, Madrid, Instituto Marxista de Economía, 720 p.

Bengoetxea, Aitor (2016): «Régimen jurídico básico de las materias directamente vinculadas a la reducción del tiempo de trabajo», Lan Harremanak, n. ${ }^{\circ}$ 34, 2016 (1).

Buendía Azorín, José Daniel y SÁnchez de la VegA, M. a del Mar (2014): «Estimación de los umbrales de crecimiento económico para la creación de empleo y reducción del desempleo con datos de panel de las provincias españolas", Comunicación a la Internacional Conference on Regional Science (disponible en http://www.reunionesdeestudios regionales.org), $20 \mathrm{p}$.

Callejo, Javier (2007): «Temporalidades y tiempo de trabajo: vivencias de trabajadores y trabajadoras», en AA.VV (Miguélez, F. y Prieto, C., dir. y coord.): Las Relaciones de Empleo en España, Siglo XXI, Madrid, 142-172.

Carrasco, Cristina (1995): «Un mundo también para nosotras», Mientras Tanto, n. ${ }^{\circ} 60$, pp. 11-19.

Carrasco Cristina, Borderías, Cristina y Torns, Teresa (2011): El trabajo de cuidados: historia, teoría y políticas, Colección «Economía Crítica \& Ecologismo Social», Madrid, CIP-Ecosocial y La Catarata, 411 p.

Castrillón Serna, Verónica, Zendoia Sainz, José Mari y Zubiri Rey, Jon Bernat (2016a): «Diagnóstico del empleo en Gipuzkoa», Lan Harremanak. Revista de Relaciones Laborales, n. ${ }^{\circ}$ 34, 2016 (1)

- (2016b): «Distribución funcional de la renta y costes laborales en Gipuzkoa: un estudio empírico para la reducción del tiempo de trabajo». Lan Harremanak. Revista de Relaciones Laborales, n. ${ }^{\circ}$ 34, 2016 (1).

- (2016c): «Indicadores socio-laborales de viabilidad por sectores de una reducción del tiempo de trabajo en Gipuzkoa», Lan Harremanak. Revista de Relaciones Laborales, n. ${ }^{\circ} 34,2016(1)$.

- (2016d): «Propuestas de reducción del tiempo de trabajo en Gipuzkoa», Lan Harremanak. Revista de Relaciones Laborales, n. ${ }^{\circ}$ 34, 2016 (1).

CES-Consejo de Relaciones Laborales (2015): Situación sociolaboral en Euskadi, II Trimestre 2015, Septiembre de 2015, 16 p.

- (1996): El trabajo a tiempo parcial, CES, Madrid.

Clavero, Vicente (2015): «Crecimiento sin empleo», Público, 1 mayo del 2015.

Coutrot, Thomas (1997): «La réduction du temps de travail: mesure technocratin que ou innovation conflictuelle?», en VV.AA., Appel des économistes pour sortir de la pensé unique. Pour un nouveau plein emploi, Paris, Alternatives Economiques-Syros.

De la Fuente Lavín, Mikel y Zubiri Rey, Jon Bernat (2016b): «Efectos sobre el empleo de la RTT: Aprendizajes a la vista de la experiencia francesa», Lan Harremanak. Revista de Relaciones Laborales, n. ${ }^{\circ}$ 34, 2016 (1).

Durán Rodríguez, José (2015): "Horas extra: el mayor robo de la historia», Diagonal, n. ${ }^{\circ} 254$, septiembre 2015,2 p. 
ELA (2015a): Análisis de coyuntura, n. ${ }^{\circ}$ 129, Manu Robles-Arangiz Institutua, septiembre 2015, $14 \mathrm{p}$.

- (2015b): Análisis de coyuntura, n. ${ }^{\circ}$ 128, Manu Robles-Arangiz Institutua, julio 2015, $12 \mathrm{p}$.

ETUi-European Trade Union Institute (2015): Benchmarking Working Europe, ETUI, Bruselas.

Eurofound (2014): Developments in collectively agreed working time 2013, disponible en http://eurofound.europa.eu

- (2010): Cambios a lo largo del tiempo. Primeras conclusiones de la quinta Encuesta europea sobre las condiciones de trabajo. Resumen, disponible en http://www.eurofound. europa.eu/

Eustat (2015): Encuesta de Población en Relación con la Actividad (EPRA), Tercer trimestre del 2015, 21 de octubre.

Fernández Domínguez, Juan José (2000): «La jornada de trabajo: duración y distribución", Social, n. ${ }^{\circ}$ 51, 23-30.

Férnandez Durán, Ramón y González Reyes, Luis (2015): En la espiral de la energía. Tomo II: Colapso del capitalismo global y civilizatorio, Madrid, Libros en Acción y Baladre, $209 \mathrm{p}$.

Fuster, Gaspar y Rodríguez Algans, Lluís (2013): «Reducción de jornada a 30 horas semanales sin reducción salarial: Análisis y perspectivas de la propuesta», 15F-Jornada de Acción contra el Paro: ii Que el paro, no te pare!!

Fita Ortega, Fernando (1999): Limites legales a la jornada de trabajo, Ed. Tirant Lo Blanch, Valencia.

Gadrey, Jean, Marcellesi, Florent y Barragué, Borja (2013): Adiós al crecimiento. Vivir bien en un mundo solidario y sostenible, EL Viejo Topo, Barcelona, $226 \mathrm{p}$.

GAINDEgia (2013): «Euskal Herriko gazteen ihesa», GIZARTEA, 13.alea, 4 orr.

García Ninet, José Ignacio (2000): «Editorial», TS.

Geroa Bai, EH Bildu, Podemos-Ahaldugu, Ezkerra (2015), Acuerdo programático para el Gobierno de Navarra. Legislatura 2015-2019, Iruñea-Pamplona, 1 de julio de 2015, 74 p.

Gilbert, Cette (1998): «Les effets d'une réduction du temps de travail sur l'emploi et le chômage», Revue française d'économie. Volume 13, N.o 3, pp. 127-149.

Hoyos Ramos, David (2006): Movilidad sostenible: hacia un concepto operativo, XVI Congreso de Estudios Vascos: Garapen Iraunkorra-IT. etorkizuna, Donostia, Eusko Ikaskuntza, pp. 327-335.

Huete, Félix (1999): «El umbral de creación de empleo en España», Cuadernos de Información Económica, núm. 148/149, 233-236.

Husson, Michel (2015): «Chômage, productivité, durée du travail», Note hussonet, n. ${ }^{\circ} 90$, 12 Octobre 2015.

Husson, Michel y Treillet, Stéphanie (2014): «La réduction du temps de travail: un combat central et d'actualité», ContreTemps, n.o 20.

Imaz BengoetxeA, José Ignacio (2006): La reducción del tiempo de trabajo, 1995-2005, Soziologiazko Euskal Koadernoak-Cuadernos Sociológicos Vascos, n. ${ }^{\circ} 21$.

- (2003): Lanaldi murrizketa eta banaketari buruzko eztabaida Euskadin, Tesia EHUn, Zuzendaria: Imanol Zubero Beaskoetxea, 527 p.

INE-Instituto Nacional de Estadística (2015a): Encuesta de Población Activa (EPA) Primer trimestre de 2015, 23 de abril de 2015, 26 p.

- (2015b): Encuesta Trimestral de Coste Laboral (ETCL), Primer trimestre de 2015, 17 de junio de 2015, 13 p. 
- (2014): «Análisis de la evolución reciente de la población activa en España (Primer trimestre de2011 a primer trimestre de 2014)", Análisis y Estudios sobre la Encuesta de Población Activa, Madrid, 22 de mayo de 2014.

Jean, Sébastien (2000): «Emploi: les enseignements de l'expérience néerlandaise», Économie et Statistique, n. ${ }^{\circ} 332-333,2000-2 / 3$, pp.153-157.

Lago Peñas, José Manuel (2013): "Un análisis cuantificado de los efectos de la reforma laboral sobre el empleo», Fundación 1o de Mayo, Colección Estudios, n. ${ }^{\circ} 61$.

Lallement, Michel (207): "Tiempo, trabajo, sujeto. Balance, cuestiones claves y perspectivas de las transformaciones contemporáneas», en AA.VV (2007) (Prieto, Carlos ed.). Trabajo, género y tiempo social, Hacer-Editorial Complutense, 49-63.

LetTIERI, Antonio (1999): "La revolución en el tiempo de trabajo», RDS, n. ${ }^{\circ} 7,31-44$.

López Hernández, Isidro y Rodríguez López, Emmanuel (2010), Financiarización, territorio y sociedad de propietarios en la honda larga del capitalismo hispano 1959-2010, Traficantes de Sueños: Útiles, n. ${ }^{\circ 9}, 513$ p.

LoRdon, Fréderic (2008): Jusqu'à quand?: Pour en finir avec les crises financières. Paris, Raison d'Agir. [2009, El porqué de las crisis financieras y cómo evitarlas, Madrid, FUHEM, Economía crítica \& ecologismo social, no 5, 2009].

MarCELLESI, Florent (2012): «Decálogo para la gran transformación ecológica», Ecología Politica, n. 44 (diciembre del 2012).

Miguélez, Fausto y Prieto, Carlos (1999) (dir. y coord.): Las Relaciones de Empleo en España, Madrid, Siglo XXI.

Monereo Pérez, José Luis, Monereo Pérez, Manuel y Ochando Claramunt, Carlos (2000): «Keynesianismo y políticas económicas y sociales: una aproximación crítica a las políticas de empleo", Sistema, n. ${ }^{\circ}$ 155-156, 71-113.

Monereo Pérez, José Luis y Medina Castillo, José Enrique (1999): «La ordenación jurídico-política del tiempo de trabajo y el reparto del empleo», Sistema, n. ${ }^{\circ} 150$, 29-50.

Montes, Pedro (1996): Golpe de estado al bienestar, Barcelona, Icaria.

Moreno Márquez, Gorka (2009): «Empleo y ciudadanía. Propuesta de debate para una relación en la encrucijada», Lan Harremanak/20-21, pp. 181-202.

- (2003): Trabajo y ciudadanía: Un debate abierto, Gasteiz, Ararteko, 371 p.

Morini, Cristina (2014): Por amor o por la fuerza. Feminización del trabajo y biopolitica del cuerpo, Madrid, Traficantes de Sueños, 223 p. Descargable en http://www. traficantes.net/

MúÑoz, Tómas (2013): "Más de 200.000 jóvenes convertidos en exiliados económicos», Diagonal, n. ${ }^{\circ} 193,18$ de marzo.

Navarro, Viçenc (1999): «¿Son las políticas socialdemócratas posibles en un país sumergido en la supuesta globalización de la economía mundial?», Sistema, n. ${ }^{\circ}$ 148, 15-27.

Oficina Internacional del Trabajo (2011): El tiempo de trabajo en el siglo XXI. Informe para el debate de la Reunión tripartita de expertos sobre la ordenación del tiempo de trabajo, OIT, Ginebra.

Orozco, Amaia Pérez (2014): Subversión feminista de la economía, Madrid, Traficantes de Sueños. Descargable en http://www.traficantes.net/

Pradella, Lucía (2015): «La carrera global a la baja», Viento Sur, 16 de septiembre, Disponible en http://vientosur.info

Prieto, Carlos y Ramos, Ramón (1999): «El tiempo de trabajo: entre la competitividad y los tiempos sociales», en AA.VV (Miguélez, Fausto y Prieto, Carlos, dir. y coord.): Las Relaciones de Empleo en España, Siglo XXI, Madrid. 
Ramírez Martínez, José Miguel (1999): «Reducción del tiempo de trabajo y reparto del empleo", Quaderns de relations laborals (Universidad de Valencia), n. ${ }^{\circ} 3$.

Riechmann, Jorge y Recio, Albert (1997): Quién parte y reparte... El debate sobre la reducción del tiempo de trabajo, Barcelona, Icaria.

Russel, Bertrand (1932): "Elogio de la ociosidad», Barcelona, Edhasa, Los libros de Sísifo, 2010, 256 p.

SchVARzer, Jorge (2004): «La apertura económica, el comercio mundial y los bloques comerciales», Disponible en http://www.uned.es/emma/schvarzer.pdf.

Soto Carmona, Álvaro (1989): El trabajo industrial en la España Contemporánea (18741936), Barcelona, Ed. Antrophos.

Tonns, Teresa (2007): "El tiempo de trabajo y las relaciones de género», en AA.VV. (2007) (Prieto, Carlos ed.): Trabajo, género y tiempo social, Hacer-Editorial Complutense, 269-278.

UGT (2015): Análisis de las horas de trabajo y la jornada laboral en Espala en el periodo 2008-2014, Gabinete Técnico Confederal, 2 de enero, 9 p.

VALVERDE, M., «La patronal reclama más horas extra y más baratas para las empresas», Expansión, 11-3-2016, 21.

VIARD, Jean (2013): «Nous en passons plus que 12\% de nôtre vie autravail», L'Obs, Disponible en http://tinyurl.com/mg9akmn

Visser, Jelle (2000), "La réduction du temps de travail aux Pays-Bas», Économie internationale, la revue du CEPII, n. ${ }^{\circ} 83$, 3éme trimestre, pp.129-152.

Zubiri Rey, Jon Bernat (2014): «El empleo juvenil como vector de transformación de las sociedades salariales de servicios», Boletin ECOS del Cip-Ecosocial, n. ${ }^{\circ} 27$, junio 2014, $15 \mathrm{p}$.

\section{Anexos}

Estas tablas son la introducción de un estudio más extenso, con numerosos cálculos sociolaborales y fiscales, realizados en el marco de la investigación sobre RTT en Gipuzkoa (Zendoia et al., 2015; Castrillón et al., 2016a; 2016b; 2016c; 2016d) 
Tabla 2

Población ocupada (miles) por sector, errialde y sexo, 1985-III - 2014-III

\begin{tabular}{|c|c|c|c|c|c|c|c|c|c|c|c|c|c|c|}
\hline \multicolumn{15}{|c|}{ Población de 16 y + años. OCUPADA. Trimestre 3 (miles) } \\
\hline $\begin{array}{c}\text { Sector } \\
\text { económico }\end{array}$ & $\begin{array}{l}\text { Territorio } \\
\text { Histórico }\end{array}$ & Sexo & 1985 & 1990 & 1995 & 2000 & 2008 & 2009 & 2010 & 2011 & 2012 & 2013 & 2014 & $\begin{array}{c}\text { Evol } \\
08-14\end{array}$ \\
\hline \multirow{8}{*}{ تृّँّ } & \multirow{2}{*}{ CAE } & Hombres & 470,7 & 503,3 & 467 & 517,6 & 564,6 & 523,4 & 523,9 & 501,4 & 483,5 & 480,7 & 474,4 & $-90,2$ \\
\hline & & Mujeres & 184,3 & 236,2 & 254,5 & 304,5 & 426,4 & 418,8 & 424,3 & 423,6 & 416,2 & 411,2 & 417,6 & $-8,8$ \\
\hline & \multirow{2}{*}{ Araba } & Hombres & 65,3 & 68,7 & 68,3 & 76,2 & 88,5 & 81,0 & 77,3 & 79,7 & 75,1 & 72,8 & 73,9 & $-14,6$ \\
\hline & & Mujeres & 27,5 & 30,3 & 37,8 & 49,4 & 63,2 & 60,9 & 64,0 & 62,3 & 61,5 & 57,7 & 61,5 & $-1,7$ \\
\hline & \multirow{2}{*}{ Bizkaia } & Hombres & 250,0 & 267,8 & 241,4 & 266,0 & 288,3 & 271,7 & 273,0 & 255,8 & 248,9 & 248,9 & 240,5 & $-47,8$ \\
\hline & & Mujeres & 89,6 & 124,7 & 135,3 & 150,1 & 221,1 & 215,9 & 220,7 & 219,2 & 215,2 & 214,4 & 214,6 & $-6,5$ \\
\hline & \multirow{2}{*}{ Gipuzkoa } & Hombres & 155,4 & 166,8 & 157,4 & 175,4 & 187,7 & 170,8 & 173,6 & 165,9 & 159,5 & 159,0 & 159,9 & $-27,8$ \\
\hline & & Mujeres & 67,2 & 81,2 & 81,4 & 104,9 & 142,1 & 141,9 & 139,6 & 142,1 & 139,5 & 139,0 & 141,5 & $-0,6$ \\
\hline \multirow{8}{*}{ 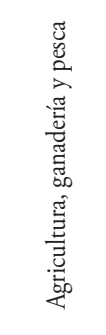 } & \multirow{2}{*}{$\mathrm{CAE}$} & Hombres & 22,9 & 17,8 & 16,6 & 12,3 & 9,1 & 7,1 & 6,7 & 5,7 & 6,6 & 6,6 & 6,6 & $-2,5$ \\
\hline & & Mujeres & 9,7 & 6,7 & 6,1 & 5,4 & 3,0 & 1,6 & 1,8 & 2,0 & 2,7 & 2,4 & 2,9 & $-0,1$ \\
\hline & \multirow{2}{*}{ Araba } & Hombres & 6,4 & 5,1 & 3,7 & 3,7 & 2,5 & 2,1 & 2,5 & 2,0 & 1,3 & 2,1 & 2,2 & $-0,3$ \\
\hline & & Mujeres & 3,2 & 1,4 & 1,7 & 1,9 & 0,8 & 0,6 & 0,8 & 0,5 & 0,8 & 1,0 & 0,9 & 0,1 \\
\hline & \multirow{2}{*}{ Bizkaia } & Hombres & 8,4 & 5,8 & 6,0 & 4,6 & 3,8 & 3,8 & 2,9 & 2,3 & 4,1 & 2,7 & 2,4 & $-1,4$ \\
\hline & & Mujeres & 3,0 & 2,8 & 2,2 & 2,0 & 1,0 & 0,4 & 0,4 & 1,2 & 1,0 & 0,6 & 0,4 & $-0,6$ \\
\hline & \multirow{2}{*}{ Gipuzkoa } & Hombres & 8,0 & 6,9 & 6,9 & 4,0 & 2,8 & 1,1 & 1,4 & 1,4 & 1,3 & 1,9 & 2,0 & $-0,8$ \\
\hline & & Mujeres & 3,6 & 2,5 & 2,2 & 1,4 & 1,2 & 0,6 & 0,6 & 0,3 & 0,8 & 0,7 & 1,6 & 0,4 \\
\hline \multirow{8}{*}{ 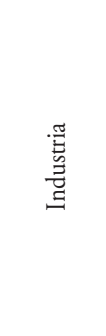 } & \multirow{2}{*}{ CAE } & Hombres & 232,8 & 223,4 & 176 & 204,7 & 205,4 & 174,6 & 181,1 & 176,5 & 168,6 & 164,9 & 157,8 & $-47,6$ \\
\hline & & Mujeres & 29,5 & 34,8 & 30,1 & 37,7 & 51,6 & 44,2 & 44,4 & 42,6 & 44,3 & 38,1 & 39,6 & $-12,0$ \\
\hline & \multirow{2}{*}{ Araba } & Hombres & 33,3 & 34,5 & 31,7 & 36,3 & 41,4 & 33,5 & 32,2 & 32,4 & 30,3 & 27,9 & 28,5 & $-12,9$ \\
\hline & & Mujeres & 5,4 & 4,5 & 5,5 & 7,1 & 9,4 & 6,8 & 7,6 & 7,5 & 8,0 & 6,2 & 5,7 & $-3,7$ \\
\hline & \multirow{2}{*}{ Bizkaia } & Hombres & 119,3 & 116,2 & 79,4 & 87,9 & 89,6 & 76 & 77,8 & 76,8 & 74,6 & 76,4 & 70,8 & $-18,8$ \\
\hline & & Mujeres & 11,4 & 15,3 & 14,5 & 14,8 & 20,8 & 18,4 & 19,4 & 18,3 & 19,9 & 16,9 & 19,4 & $-1,4$ \\
\hline & \multirow{2}{*}{ Gipuzkoa } & Hombres & 80,2 & 72,8 & 64,8 & 80,5 & 74,5 & 65,1 & 71,1 & 67,3 & 63,7 & 60,5 & 58,5 & $-16,0$ \\
\hline & & Mujeres & 12,8 & 15,0 & 10,1 & 15,9 & 21,3 & 19,0 & 17,5 & 16,7 & 16,5 & 15,1 & 14,5 & $-6,8$ \\
\hline \multirow{8}{*}{ 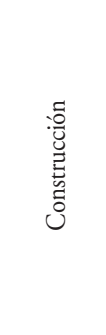 } & \multirow{2}{*}{$\mathrm{CAE}$} & Hombres & 38,1 & 53 & 56,6 & 70,2 & 71,6 & 70,0 & 65,0 & 60,0 & 50,7 & 39,3 & 42,9 & $-28,7$ \\
\hline & & Mujeres & 0,3 & 4,1 & 2,6 & 3,2 & 7,9 & 7,4 & 7,5 & 6,7 & 6,6 & 5,9 & 4,8 & $-3,1$ \\
\hline & \multirow{2}{*}{ Araba } & Hombres & 5,6 & 6,7 & 7,7 & 9,0 & 8,5 & 8,6 & 8,0 & 8,5 & 5,9 & 6,0 & 6,3 & $-2,2$ \\
\hline & & Mujeres & - & 0,4 & 0,4 & 0,3 & 0,7 & 0,7 & 1,1 & 1,8 & 1,0 & 0,4 & 0,7 & 0,0 \\
\hline & \multirow{2}{*}{ Bizkaia } & Hombres & 21,5 & 28,9 & 33,2 & 41,2 & 38,3 & 39,3 & 38,4 & 31,6 & 27,9 & 21,5 & 23,3 & -15 \\
\hline & & Mujeres & 0,3 & 3,1 & 1,2 & 1,7 & 4,7 & 4,5 & 4,2 & 3,0 & 2,7 & 3,4 & 3,0 & $-1,7$ \\
\hline & \multirow{2}{*}{ Gipuzkoa } & Hombres & 10,9 & 17,4 & 15,7 & 20 & 24,8 & 22,2 & 18,6 & 19,9 & 16,9 & 11,8 & 13,3 & $-11,5$ \\
\hline & & Mujeres & - & 0,6 & 1,0 & 1,2 & 2,4 & 2,2 & 2,3 & 1,8 & 2,9 & 2,1 & 1,2 & $-1,2$ \\
\hline & CAF & Hombres & 176,9 & 209,0 & 217,9 & 230,4 & 278,4 & 271,7 & 271,1 & 259,2 & 257,6 & 269,8 & 267,1 & $-11,3$ \\
\hline & CAE & Mujeres & 144,8 & 190,7 & 215,6 & 258,2 & 363,9 & 365,5 & 370,6 & 372,3 & 362,5 & 364,8 & 370,2 & 6,3 \\
\hline & Araba & Hombres & 20,0 & 22,3 & 25,2 & 27,2 & 36,1 & 36,7 & 34,6 & 36,8 & 37,7 & 36,8 & 37,0 & 0,9 \\
\hline$\stackrel{\mathscr{0}}{\underline{\sigma}}$ & Arada & Mujeres & 18,9 & 23,9 & 30,1 & 40,1 & 52,3 & 52,8 & 54,7 & 52,5 & 51,6 & 50,2 & 54,1 & 1,8 \\
\hline ڤ్ & Bizkiz & Hombres & 100,7 & 116,9 & 122,7 & 132,3 & 156,6 & 152,5 & 153,9 & 145,2 & 142,3 & 148,3 & 144 & $-12,6$ \\
\hline & Dizkala & Mujeres & 75,0 & 103,6 & 117,4 & 131,7 & 194,5 & 192,6 & 196,7 & 196,6 & 191,7 & 193,5 & 191,8 & $-2,7$ \\
\hline & Ginurkor & Hombres & 56,2 & 69,8 & 69,9 & 70,9 & 85,7 & 82,5 & 82,5 & 77,2 & 77,6 & 84,8 & 86 & 0,3 \\
\hline & Glpuzkoa & Mujeres & 50,9 & 63,1 & 68,1 & 86,4 & 117,1 & 120,1 & 119,2 & 123,2 & 119,3 & 121,1 & 124,2 & 7,1 \\
\hline
\end{tabular}

Fuente: EPRA (Eustat, 2015). 
Tabla 3

Población de 16 y más años asalariada indefinida y temporal por errialdes y por sexos (en miles), 1993-III - 2014-III

\begin{tabular}{|c|c|c|c|c|c|c|c|c|c|c|c|c|c|}
\hline \multicolumn{14}{|c|}{ Población de 16 y + años asalariada ( $3{ }^{\text {er }}$ trimestre) (miles) } \\
\hline $\begin{array}{l}\text { Tipo de } \\
\text { contrato }\end{array}$ & $\begin{array}{l}\text { Territorio } \\
\text { Histórico }\end{array}$ & Sexo & 1993 & 1997 & 2001 & 2008 & 2009 & 2010 & 2011 & 2012 & 2013 & 2014 & $\begin{array}{c}\text { Evol } \\
08-14\end{array}$ \\
\hline \multirow{8}{*}{ 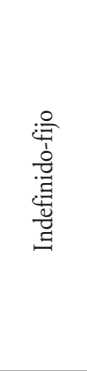 } & \multirow{2}{*}{ CAE } & Hombres & 281,9 & 249,7 & 302,6 & 370,7 & 347,8 & 340,3 & 323,5 & 306,1 & 289 & 273,5 & $-97,2$ \\
\hline & & Mujeres & 113,6 & 113,4 & 167,3 & 266,5 & 265,2 & 265,9 & 264,6 & 258,6 & 246,3 & 246,4 & $-20,1$ \\
\hline & \multirow{2}{*}{ Araba } & Hombres & 42,1 & 38,8 & 51,5 & 62,2 & 58,0 & 56,5 & 54,0 & 51,7 & 47,6 & 46,2 & $-16,0$ \\
\hline & & Mujeres & 15,3 & 17,2 & 25,2 & 42,3 & 42,0 & 44,1 & 43,4 & 40,2 & 35,7 & 37,5 & $-4,8$ \\
\hline & \multirow{2}{*}{ Bizkaia } & Hombres & 147,6 & 133,4 & 156,2 & 193,8 & 182,7 & 179,4 & 173,4 & 159,7 & 145,9 & 136,3 & $-57,5$ \\
\hline & & Mujeres & 58,4 & 61,7 & 89,4 & 143 & 143,7 & 141,8 & 139 & 136,2 & 128,9 & 126,5 & $-16,5$ \\
\hline & \multirow{2}{*}{ Gipuzkoa } & Hombres & 92,2 & 77,6 & 94,9 & 114,7 & 107,1 & 104,4 & 96,1 & 94,7 & 95,4 & 91,1 & $-23,6$ \\
\hline & & Mujeres & 40,0 & 34,5 & 52,7 & 81,2 & 79,5 & 80,0 & 82,2 & 82,3 & 81,6 & 82,4 & 1,2 \\
\hline \multirow{8}{*}{ 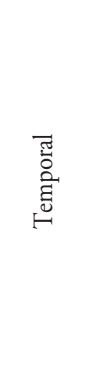 } & \multirow{2}{*}{ CAE } & Hombres & 74,3 & 105,7 & 109,2 & 85,7 & 68,5 & 74,4 & 75,4 & 73,4 & 72,6 & 81,4 & $-4,3$ \\
\hline & & Mujeres & 50,7 & 69,7 & 91,7 & 84,1 & 77,8 & 87,6 & 85,8 & 89,6 & 88,8 & 90,8 & 6,7 \\
\hline & \multirow{2}{*}{ Araba } & Hombres & 9,5 & 18,8 & 10,9 & 12,1 & 9,2 & 7,6 & 11,1 & 10,0 & 9,5 & 12,3 & 0,2 \\
\hline & & Mujeres & 10,1 & 12,4 & 13,2 & 13,6 & 11,4 & 12,1 & 12,2 & 14,1 & 13,9 & 14,6 & 1,0 \\
\hline & \multirow{2}{*}{ Bizkaia } & Hombres & 45,2 & 50,5 & 64,8 & 44,1 & 37,3 & 44,0 & 39,1 & 40,8 & 41,6 & 41,0 & $-3,1$ \\
\hline & & Mujeres & 29,6 & 33,8 & 46,1 & 40,7 & 33,8 & 44,1 & 42,1 & 47,4 & 45,5 & 46,2 & 5,5 \\
\hline & \multirow{2}{*}{ Gipuzkoa } & Hombres & 19,6 & 36,4 & 33,6 & 29,5 & 21,9 & 22,8 & 25,2 & 22,6 & 21,5 & 28,1 & $-1,4$ \\
\hline & & Mujeres & 11,1 & 23,5 & 32,4 & 29,7 & 32,6 & 31,4 & 31,5 & 28,1 & 29,5 & 30,0 & 0,3 \\
\hline \multirow{8}{*}{ 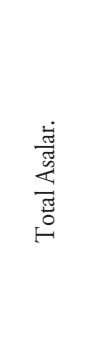 } & \multirow{2}{*}{ CAE } & Hombres & 356,2 & 355,4 & 411,8 & 456,4 & 416,3 & 414,7 & 398,9 & 379,5 & 361,6 & 354,9 & $-101,5$ \\
\hline & & Mujeres & 164,3 & 183,1 & 259 & 350,6 & 343 & 353,5 & 350,4 & 348,2 & 335,1 & 337,2 & $-13,4$ \\
\hline & \multirow{2}{*}{ Araba } & Hombres & 51,6 & 57,6 & 62,4 & 74,3 & 67,2 & 64,1 & 65,1 & 61,7 & 57,1 & 58,5 & $-15,8$ \\
\hline & & Mujeres & 25,4 & 29,6 & 38,4 & 55,9 & 53,4 & 56,2 & 55,6 & 54,3 & 49,6 & 52,1 & $-3,8$ \\
\hline & \multirow{2}{*}{ Bizkaia } & Hombres & 192,8 & 183,9 & 221 & 237,9 & 220 & 223,4 & 212,5 & 200,5 & 187,5 & 177,3 & $-60,6$ \\
\hline & & Mujeres & 88,0 & 95,5 & 135,5 & 183,7 & 177,5 & 185,9 & 181,1 & 183,6 & 174,4 & 172,7 & $-11,0$ \\
\hline & \multirow{2}{*}{ Gipuzkoa } & Hombres & 111,8 & 114 & 128,5 & 144,2 & 129 & 127,2 & 121,3 & 117,3 & 116,9 & 119,2 & $-25,0$ \\
\hline & & Mujeres & 51,1 & 58,0 & 85,1 & 110,9 & 112,1 & 111,4 & 113,7 & 110,4 & 111,1 & 112,4 & 1,5 \\
\hline
\end{tabular}

Fuente: EPRA (Eustat, 2015). 


\section{Tabla 4}

Población de 16 y más, Total, ocupada, parada e inactiva (en miles) por errialde y por sexo, 1985-III - 2015-III

\begin{tabular}{|c|c|c|c|c|c|c|c|c|c|c|c|c|c|c|}
\hline \multicolumn{15}{|c|}{ Población de $16 \mathrm{y}+$ (miles). CAE. Trimestre 3} \\
\hline $\begin{array}{l}\text { EPRA } \\
\text { (OIT) }\end{array}$ & $\begin{array}{l}\text { Territorio } \\
\text { Histórico }\end{array}$ & Sexo & 1985 & 1995 & 2005 & 2008 & 2009 & 2010 & 2011 & 2012 & 2013 & 2014 & 2015 & $\begin{array}{c}\text { Evol } \\
08-15\end{array}$ \\
\hline \multirow{8}{*}{ 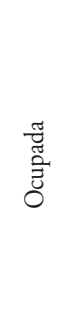 } & \multirow{2}{*}{ CAE } & Hombres & 470,7 & 467 & 557,2 & 564,6 & 523,4 & 523,9 & 501,4 & 483,5 & 480,7 & 474,4 & 467,2 & $-97,4$ \\
\hline & & Mujeres & 184,3 & 254,5 & 394,6 & 426,4 & 418,8 & 424,3 & 423,6 & 416,2 & 411,2 & 417,6 & 418,5 & $-7,9$ \\
\hline & \multirow{2}{*}{ Araba } & Hombres & 65,3 & 68,3 & 85,3 & 88,5 & 81,0 & 77,3 & 79,7 & 75,1 & 72,8 & 73,9 & 73,9 & $-14,6$ \\
\hline & & Mujeres & 27,5 & 37,8 & 59,9 & 63,2 & 60,9 & 64,0 & 62,3 & 61,5 & 57,7 & 61,5 & 60,4 & $-2,8$ \\
\hline & \multirow{2}{*}{ Bizkaia } & Hombres & 250 & 241,4 & 284,7 & 288,3 & 271,7 & 273 & 255,8 & 248,9 & 248,9 & 240,5 & 237,0 & $-51,3$ \\
\hline & & Mujeres & 89,6 & 135,3 & 202 & 221,1 & 215,9 & 220,7 & 219,2 & 215,2 & 214,4 & 214,6 & 221,3 & 0,2 \\
\hline & \multirow{2}{*}{ Gipuzkoa } & Hombres & 155,4 & 157,4 & 187,1 & 187,7 & 170,8 & 173,6 & 165,9 & 159,5 & 159,0 & 159,9 & 156,3 & $-31,4$ \\
\hline & & Mujeres & 67,2 & 81,4 & 132,8 & 142,1 & 141,9 & 139,6 & 142,1 & 139,5 & 139,0 & 141,5 & 136,7 & $-5,4$ \\
\hline \multirow{8}{*}{ 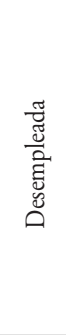 } & \multirow{2}{*}{ CAE } & Hombres & 92,9 & 94,3 & 24,9 & 19,2 & 52,6 & 54,6 & 63,7 & 63,6 & 83,9 & 82,2 & 80,3 & 61,1 \\
\hline & & Mujeres & 88,7 & 120,6 & 24,6 & 17,0 & 32,2 & 36,4 & 45,6 & 51,6 & 69,2 & 69,6 & 72,0 & 55,0 \\
\hline & \multirow{2}{*}{ Araba } & Hombres & 9,2 & 11,3 & 1,6 & 0,9 & 8,0 & 8,3 & 4,5 & 8,2 & 12,7 & 12,6 & 10,5 & 9,6 \\
\hline & & Mujeres & 8,9 & 16,3 & 1,9 & 1,0 & 7,7 & 6,4 & 6,0 & 6,9 & 13,2 & 12,9 & 12,6 & 11,6 \\
\hline & \multirow{2}{*}{ Bizkaia } & Hombres & 53,7 & 55,8 & 18,9 & 12,4 & 29,9 & 33,4 & 44,5 & 39,6 & 49,0 & 47,6 & 47,7 & 35,3 \\
\hline & & Mujeres & 50,0 & 66,0 & 15,5 & 11,7 & 17,4 & 20,3 & 29,2 & 33,3 & 37,6 & 36,5 & 40,9 & 29,2 \\
\hline & \multirow{2}{*}{ Gipuzkoa } & Hombres & 30,1 & 27,2 & 4,4 & 5,9 & 14,7 & 12,9 & 14,7 & 15,8 & 22,2 & 22,0 & 22,1 & 16,2 \\
\hline & & Mujeres & 29,8 & 38,3 & 7,2 & 4,3 & 7,0 & 9,7 & 10,3 & 11,3 & 18,3 & 20,2 & 18,5 & 14,2 \\
\hline & & & & & & & & & & & & & & $\begin{array}{c}\text { Evol } \\
08-14\end{array}$ \\
\hline \multirow{8}{*}{ 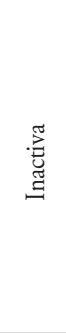 } & \multirow{2}{*}{ CAE } & Hombres & 232,2 & 303,7 & 305,9 & 319,1 & 326,1 & 320,3 & 333,5 & 347,5 & 322,9 & 327,1 & 776,9 (2015-III) & 8,0 \\
\hline & & Mujeres & 557,7 & 536,7 & 518,8 & 509,3 & 502,8 & 491,2 & 485,1 & 484,3 & 469,9 & 458,8 & 785,9 (2014-III) & $-50,5$ \\
\hline & \multirow{2}{*}{ Araba } & Hombres & 24,6 & 36,4 & 42,1 & 44,2 & 45,5 & 49,2 & 50,8 & 50,4 & 46,8 & 45,5 & & 1,3 \\
\hline & & Mujeres & 63,1 & 64,4 & 68,6 & 70,3 & 66,6 & 65,4 & 68,0 & 67,9 & 65,3 & 61,4 & & $-8,9$ \\
\hline & \multirow{2}{*}{ Bizkaia } & Hombres & 136,8 & 172,4 & 170,5 & 177,9 & 176,1 & 169,3 & 174,3 & 183,2 & 169,4 & 176,8 & & $-1,1$ \\
\hline & & Mujeres & 323,3 & 298,7 & 292,2 & 282,5 & 281,9 & 272,1 & 265,4 & 263,9 & 258,6 & 256,9 & & $-25,6$ \\
\hline & \multirow{2}{*}{ Gipuzkoa } & Hombres & 70,8 & 95,0 & 93,3 & 97,0 & 104,5 & 101,9 & 108,5 & 113,9 & 106,8 & 104,8 & & 7,8 \\
\hline & & Mujeres & 171,2 & 173,7 & 158,1 & 156,5 & 154,4 & 153,7 & 151,7 & 152,4 & 145,9 & 140,6 & & $-15,9$ \\
\hline \multirow{8}{*}{ 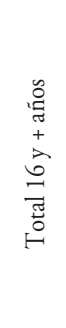 } & \multirow{2}{*}{ CAE } & Hombres & 795,8 & 865,0 & 888,0 & 902,8 & 902,2 & 898,9 & 898,6 & 894,5 & 887,5 & 883,7 & & $-19,1$ \\
\hline & & Mujeres & 830,7 & 911,8 & 938,1 & 952,6 & 953,8 & 952,0 & 954,2 & 952,1 & 950,2 & 946,0 & & $-6,6$ \\
\hline & \multirow{2}{*}{ Araba } & Hombres & 99,1 & 116,0 & 129,0 & 133,7 & 134,5 & 134,8 & 134,9 & 133,7 & 132,2 & 132,1 & & $-1,6$ \\
\hline & & Mujeres & 99,6 & 118,4 & 130,3 & 134,5 & 135,3 & 135,8 & 136,3 & 136,3 & 136,3 & 135,8 & & 1,3 \\
\hline & \multirow{2}{*}{ Bizkaia } & Hombres & 440,4 & 469,5 & 474,1 & 478,6 & 477,7 & 475,7 & 474,6 & 471,7 & 467,3 & 464,9 & & $-13,7$ \\
\hline & & Mujeres & 462,9 & 500 & 509,7 & 515,3 & 515,2 & 513,1 & 513,9 & 512,4 & 510,6 & 508,0 & & $-7,3$ \\
\hline & \multirow{2}{*}{ Gipuzkoa } & Hombres & 256,3 & 279,5 & 284,8 & 290,6 & 290 & 288,4 & 289,1 & 289,1 & 287,9 & 286,7 & & $-3,9$ \\
\hline & & Mujeres & 268,3 & 293,4 & 298,1 & 302,9 & 303,3 & 303,1 & 304,0 & 303,3 & 303,2 & 302,2 & & $-0,7$ \\
\hline
\end{tabular}

Fuente: EPRA (Eustat, 2015). 
Tabla 5

Variaciones absolutas de Ocupados, Parados, Inactivos y Totales (en miles) por errialde y por sexo, 2008-III - 2014-III

\begin{tabular}{|c|c|c|c|c|c|}
\hline & & \multicolumn{4}{|c|}{ Variaciones Absolutas 2008-III - 2014-III } \\
\hline & & Var. Ocups. & Var. Parads. & Var. Inactivs & Var. Total \\
\hline \multirow{2}{*}{ CAE } & Hombres & $-90,2$ & 63,0 & 8,0 & $-19,1$ \\
\hline & Mujeres & $-8,8$ & 52,6 & $-50,5$ & $-6,6$ \\
\hline \multirow{2}{*}{ Araba } & Hombres & $-14,6$ & 11,7 & 1,3 & $-1,6$ \\
\hline & Mujeres & $-1,7$ & 11,9 & $-8,9$ & 1,3 \\
\hline \multirow{2}{*}{ Bizkaia } & Hombres & $-47,8$ & 35,2 & $-1,1$ & $-13,7$ \\
\hline & Mujeres & $-6,5$ & 24,8 & $-25,6$ & $-7,3$ \\
\hline \multirow{2}{*}{ Gipuzkoa } & Hombres & $-27,8$ & 16,1 & 7,8 & $-3,9$ \\
\hline & Mujeres & $-0,6$ & 15,9 & $-15,9$ & $-0,7$ \\
\hline
\end{tabular}

Var. Total $(>16)$ = Var. Ocup.+Var. Parad.+Var. Inact.

Fuente: EPRA (Eustat, 2015).

Tabla 6

Indicadores sociolaborales en Gipuzkoa, 1995-2007

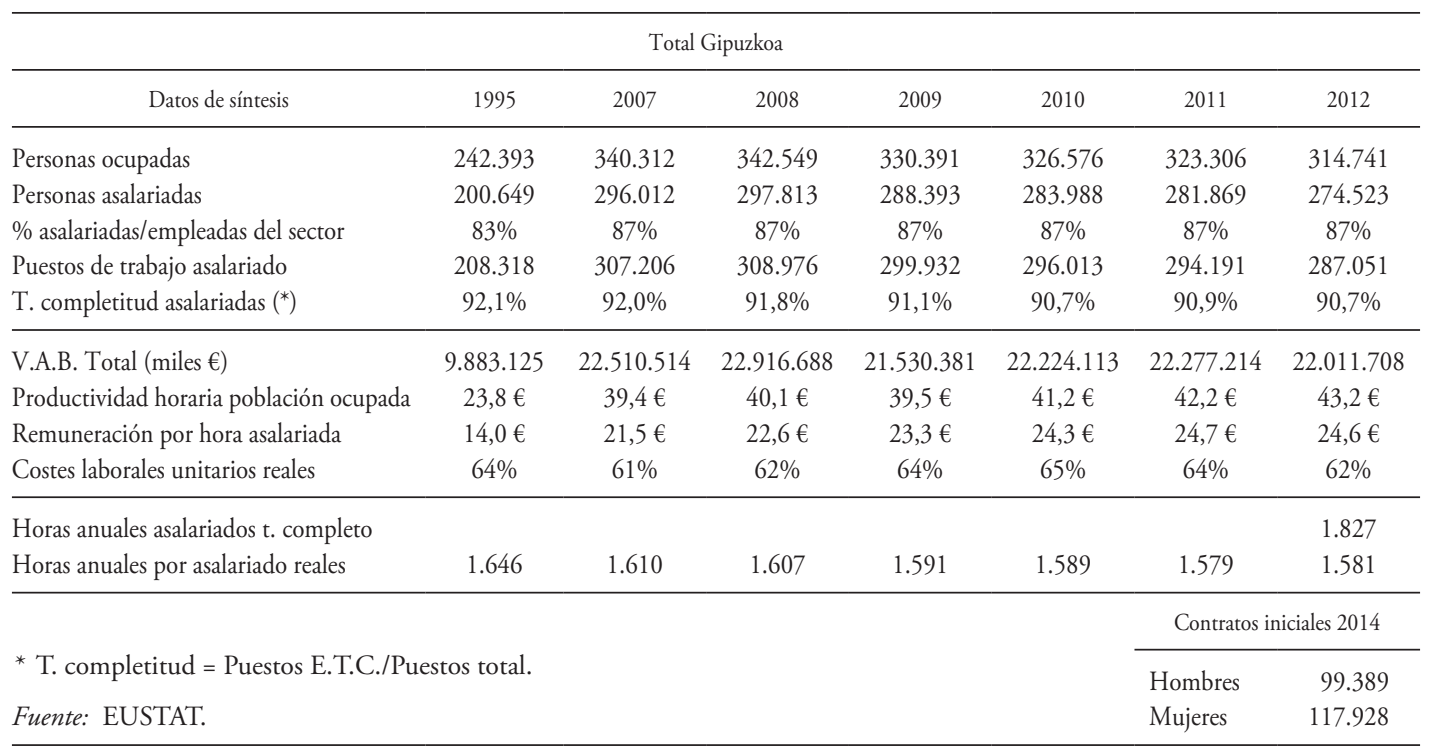

Fuente: Castrillón et al. (2016c). 